\title{
Inclusive diffraction in future electron-proton and electron-ion colliders
}

\author{
Néstor Armesto $\odot,{ }^{1}$ Paul R. Newman, ${ }^{2}$ Wojciech Słomiński® ${ }^{3}$ and Anna M. Staśto ${ }^{4}$ \\ ${ }^{1}$ Instituto Galego de Física de Altas Enerxías IGFAE, Universidade de Santiago de Compostela, \\ 15782 Santiago de Compostela, Galicia-Spain \\ ${ }^{2}$ School of Physics and Astronomy, University of Birmingham, B15 2TT, United Kingdom \\ ${ }^{3}$ Institute of Physics, Jagiellonian University, 30-348 Krakow, Poland \\ ${ }^{4}$ Department of Physics, Penn State University, University Park, Pennsylvania 16802, USA
}

(Received 29 August 2019; published 21 October 2019)

\begin{abstract}
We analyze the possibilities for the study of inclusive diffraction offered by future electron-proton/ nucleus colliders in the tera-electron-volt regime, the Large Hadron-electron Collider (LHeC) as an upgrade of the HL-LHC, and the Future Circular Collider in electron-hadron mode. Compared to $e p$ collisions at HERA, we find an extension of the available kinematic range in $x$ by a factor of order 20 and of the maximum $Q^{2}$ by a factor of order 100 for $\mathrm{LHeC}$, while the Future Circular Collider (FCC) version would extend the coverage by a further order of magnitude both in $x$ and $Q^{2}$. This translates into a range of the available momentum fraction of the diffractive exchange with respect to the hadron $(\xi)$, down to $10^{-4}-10^{-5}$ for a wide range of the momentum fraction of the parton with respect to the diffractive exchange $(\beta)$. Using the same framework and methodology employed in previous studies at HERA, considering only the experimental uncertainties and not those stemming from the functional form of the initial conditions or other ones of theoretical origin, and under very conservative assumptions for the luminosities and systematic errors, we find an improvement in the extraction of diffractive parton densities from fits to reduced cross sections for inclusive coherent diffraction in $e p$ by about an order of magnitude. For $e A$, we also perform the simulations for the Electron Ion Collider. We find that an extraction of the currently unmeasured nuclear diffractive parton densities is possible with accuracy similar to that in $e p$.
\end{abstract}

DOI: 10.1103/PhysRevD.100.074022

\section{INTRODUCTION}

Deep inelastic scattering (DIS) of a lepton on a proton is the cleanest way to explore the proton structure. The HERA accelerator in Hamburg has been the only $e p$ collider to date. It scattered electrons and positrons on protons, at center-of-mass energy $\sqrt{s}=318 \mathrm{GeV}$. One of the most striking discoveries at HERA was the observation of the strong rise of the gluon density at small values of Bjorken $x$. HERA provided the measurement of the parton densities with high accuracy, necessary for precise theoretical calculations of a vast range of processes under study at the LHC. Another discovery of HERA was the observation of a large (approximately 10\%) fraction of diffractive events in DIS [1,2]; see the review [3] and references therein. In these events, the proton stays intact or dissociates into a state with the proton quantum numbers, despite undergoing a violent, highly energetic collision, and is

Published by the American Physical Society under the terms of the Creative Commons Attribution 4.0 International license. Further distribution of this work must maintain attribution to the author(s) and the published article's title, journal citation, and DOI. Funded by SCOAP ${ }^{3}$. separated from the rest of the produced particles by a large rapidity gap. In a series of ground-breaking papers, the HERA experiments determined the deep inelastic structure of the $t$-channel exchange in these events in the form of diffractive parton densities.

The precise measurement of diffraction in DIS is of great importance for our understanding of the strong interaction. First, the mechanism through which a composite strongly interacting object interacts perturbatively while keeping color neutrality offers information about the confinement mechanism. Second, diffraction is known to be highly sensitive to the low- $x$ partonic content of the proton and its evolution with energy, and it therefore has considerable promise to reveal deviations from standard linear evolution through higher-twist effects or, eventually, nonlinear dynamics. Third, it allows checks of basic theory predictions such as the relation between diffraction in $e p$ scattering and nuclear shadowing [4]. Finally, the accurate extraction of diffractive parton distribution functions (PDFs) facilitates tests of the range of validity of the perturbative factorization [5-7]. We note that our study is only about inclusive diffraction. Other aspects could be addressed, for example factorization breaking in diffractive dijet photoproduction [8,9], which can also be studied in ultraperipheral collisions at the LHC [10]. 
Diffraction has also been studied outside the collinear framework, for example in the color glass condensate [11] and its implementation in photoproduction and DIS through the dipole model $[12,13]$. Differences are expected with respect to collinear factorization at small $x$, resulting in particularly strong modifications of diffraction in nuclei with respect to the proton [14,15]; see the detailed discussions in Ref. [16].

Future DIS machines could explore this phenomenon at higher energies and with much higher precision. The Large Hadron-electron Collider (LHeC) is a proposal [17-19] for an $e p$ and $e A$ machine at CERN. It would utilize the $7 \mathrm{TeV}$ proton beam from the LHC and collide it with a $60 \mathrm{GeV}$ electron beam accelerated by an energy recovery linac, thus reaching a center-of-mass energy $\sqrt{s}=1.3 \mathrm{TeV}$. Dedicated studies of the machine parameters $[20,21]$ show that its peak luminosity would reach $10^{34} \mathrm{~cm}^{-2} \mathrm{~s}^{-1}$, about 3 orders of magnitude higher than HERA. The projected running of the machine is over three periods. In the initial run period, the total integrated luminosity is estimated to be $50 \mathrm{fb}^{-1}$. Throughout the entire operation, the $\mathrm{LHeC}$ is projected to reach $1 \mathrm{ab}^{-1}$ integrated luminosity. It would also be the first electron-nucleus collider, as it would scatter electrons on a beam of nuclei from the LHC, with an energy of $2.75 \mathrm{TeV}$ per nucleon resulting in the center-of-mass energy per nucleon $\sqrt{s}=812 \mathrm{GeV}$. The integrated luminosity for collisions on nuclei is projected to be of the order $10 \mathrm{fb}^{-1}$, which is ten times larger than the total luminosity collected in $e p$ at HERA. This would allow measurements of nuclear structure with unprecedented precision. Beyond $\mathrm{LHeC}$, the next generation $e p$ collider would be the Future Circular Collider in electron-hadron mode (FCC-eh), utilizing the $50 \mathrm{TeV}$ proton beam from the FCC $[22,23]$ which would probe DIS at a center-of-mass energy of $\sqrt{s}=$ $3.5 \mathrm{TeV}$ with a total integrated luminosity of several $\mathrm{ab}^{-1}$. The $e A$ collisions at the FCC-eh $[20,21]$ would be performed with a lead beam with energy per nucleon 19.7 TeV, which would give a center-of-mass energy per nucleon of $\sqrt{s}=2.2 \mathrm{TeV}$. At lower energies $\sqrt{s} \sim 0.1 \mathrm{TeV}$, the Electron Ion Collider (EIC) [24] will also measure diffraction, covering a smaller kinematic region than HERA in $e p$ but a completely novel region in $e$ A with respect to fixed target experiments where diffraction has been barely studied.

These machines would facilitate the study of the proton and nuclear structure with extremely high precision. They would unravel complete details of the partonic structure of the proton, explore novel QCD dynamics at small values of Bjorken $x$, constrain the Higgs properties, perform searches for physics beyond the Standard Model, and provide complementary precision measurements of electroweak physics to $e^{+} e^{-}$colliders and the LHC and FCC-hh. DIS on nuclei would allow the study of nuclear structure in a previously unexplored kinematic region in $\left(x, Q^{2}\right)$. It is therefore expected to thoroughly transform our present knowledge on parton structure in nuclei, also largely strengthening the chromodynamic base for the quark gluon plasma and the ridge correlation phenomenon.

In this work, we perform a thorough analysis of the capability of the LHeC and FCC-eh machines to explore inclusive diffraction in DIS. We first determine the accessible kinematic range for diffraction of both machines. Using a very conservative assumption of $2 \mathrm{fb}^{-1}$ for the integrated luminosity, we perform a simulation of the data for inclusive coherent $e p$ diffraction in the projected parameter space. This is performed by extending the fits used to extract the diffractive parton densities (DPDFs) at HERA. We then demonstrate the potential of both machines to constrain the DPDFs and point out the sensitivity to the interesting region of low $Q^{2}$ where deviations from standard linear evolution could become sizeable. These machines would also be able to explore the top-quark contribution to diffraction as well as measure diffraction in the charged current exchange, though we do not perform analysis of these interesting phenomena here. We analyze the sensitivity to kinematic cuts and variations of the fit framework. We also note the possibilities that measurements at these new machines offer of improving existing constraints on the shape of the gluon distribution and the sensitivity to physics beyond linear twist-2 DokshitzerGribov-Lipatov-Altarelli-Parisi evolution at moderate $Q^{2}$.

We also perform a simulation of the diffractive pseudodata for $e A$ collisions for different scenarios of nuclear shadowing. Nuclear diffractive parton distributions have never been measured, and therefore the considered machines would be the first to extract these important quantities. It would also be possible to investigate the relation between nuclear shadowing and diffraction.

We focus on the impact of the new kinematic range and expected increase in the measurement accuracy on the extraction of diffractive parton densities from fits to cross sections for inclusive coherent diffraction in $e p$. To this end, we stick to the parametrization model used in the HERA fits, and we estimate the experimental uncertainties of DPDFs obtained from fits to pseudodata generated for the $\mathrm{LHeC} / \mathrm{FCC}$-eh scenario. Comparing these uncertainties to the ones resulting from the HERA data, we observe an order of magnitude improvement. It should be noted that the values of these DPDF uncertainties come from the experimental uncertainties only, and as such, they are most likely below the expected full uncertainties which would also arise from the parametrization and theory uncertainties. Nevertheless, the relative improvement clearly shows the measurement potential of the new machines.

The structure of the paper is the following. In Sec. II, we recall the formulas for the diffractive cross sections, the factorization of the inclusive diffractive structure functions, and the origin of their sensitivity to DPDFs. In Sec. III, we present the details of the simulations for the diffractive DIS. In particular, in Sec. III A, we discuss the parametrization 
used at HERA; in Sec. III B, we show the details of the diffractive kinematic range in new machines; and in Sec. III C, the method to obtain the projected pseudodata with errors is discussed. In Sec. IV, we present our fitting methodology and the potential for constraining the diffractive parton densities by both machines. Section V is devoted to the prospects of the diffractive deep inelastic in nuclei. Finally, we summarize our findings in Sec. VI.

\section{DIFFRACTIVE CROSS SECTION AND DIFFRACTIVE PDFs}

In Fig. 1, we show a diagram depicting a neutral current diffractive deep inelastic event. Charged currents could also be considered, and they were measured at HERA [25] but with large statistical uncertainties and in a very restricted region of phase space. Although they could be measured at both the $\mathrm{LHeC}$ and the FCC-eh with larger statistics and more extended kinematics, in this first study, we limit ourselves to neutral currents. The incoming electron or positron, with 4-momentum $k$, scatters off the proton, with incoming momentum $p$, and the interaction proceeds through the exchange of a virtual photon with 4-momentum $q$. The kinematic variables for such an event include the standard deep inelastic variables

$$
Q^{2}=-q^{2}, \quad x=\frac{-q^{2}}{2 p \cdot q}, \quad y=\frac{p \cdot q}{p \cdot k},
$$

where $Q^{2}$ describes the photon virtuality, $x$ is the Bjorken variable, and $y$ the inelasticity of the process. In addition, the variables

$$
s=(k+p)^{2}, \quad W^{2}=(q+p)^{2}
$$

are the electron-proton center-of-mass energy squared and the photon-proton center-of-mass energy squared, respectively. The distinguishing feature of the diffractive event $e p \rightarrow e X Y$ is the presence of the large rapidity gap between the diffractive system, characterized by the invariant mass

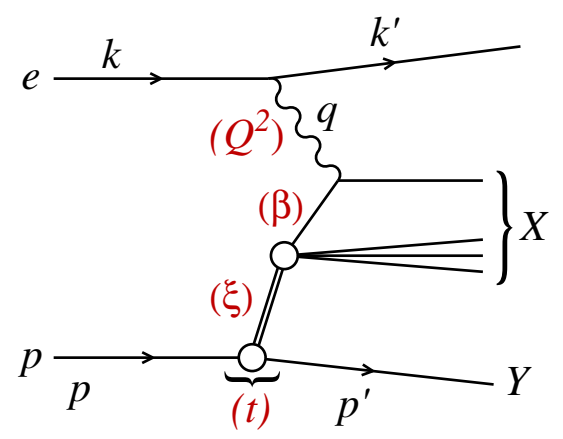

FIG. 1. A diagram of a diffractive neutral current (NC) event in DIS together with the corresponding variables, in the one-photon exchange approximation. The large rapidity gap is between the system $X$ and the scattered proton $Y$ (or its low-mass excitation).
$M_{X}$ and the final proton (or its low-mass excitation) $Y$ with 4-momentum $p^{\prime}$. In addition to the standard DIS variables listed above, diffractive events are also characterized by an additional set of variables defined as

$t=\left(p-p^{\prime}\right)^{2}, \quad \xi=\frac{Q^{2}+M_{X}^{2}-t}{Q^{2}+W^{2}}, \quad \beta=\frac{Q^{2}}{Q^{2}+M_{X}^{2}-t}$.

In the above, $t$ is the squared 4-momentum transfer at the proton vertex, $\xi$ (alternatively denoted by $x_{\mathbb{P}}$ ) can be interpreted as the momentum fraction of the "diffractive exchange" with respect to the hadron, and $\beta$ is the momentum fraction of the parton with respect to the diffractive exchange. The two momentum fractions combine to give Bjorken $x, x=\beta \xi$.

The physical picture suggested by Fig. 1 is that the initial proton splits into a final state $Y$ of momentum $p^{\prime} \simeq(1-\xi) p$ and the object which is responsible for the diffractive exchange of momentum $\xi p$. The latter in turn undergoes a DIS-like process to produce the final state $X$ (see Sec. III A for more details). The study presented in this paper concerns coherent diffraction (i.e., the nondissociating case), where the final state $Y$ is a proton. Experimentally, this requires tagging of the final proton, which was performed at HERA using Roman pot insertions to the forward beam pipe, for example the FPS (LPS) of the H1 (ZEUS) collaborations. Most of the HERA data are based, however, on the large rapidity gap (LRG) technique, which results in a small proton dissociative admixture- the response from detector components at very forward rapidities, supplemented with dedicated Monte Carlo (MC) modeling, were used to normalize these results to the coherent cross sections $[25,26]$.

Diffractive cross sections in the neutral current case can be presented in the form of the reduced cross sections [25]

$$
\frac{d^{4} \sigma^{\mathrm{D}}}{d \xi d \beta d Q^{2} d t}=\frac{2 \pi \alpha_{\mathrm{em}}^{2}}{\beta Q^{4}} Y_{+} \sigma_{\mathrm{r}}^{\mathrm{D}(4)}
$$

or, upon integration over $t$,

$$
\frac{d^{3} \sigma^{\mathrm{D}}}{d \xi d \beta d Q^{2}}=\frac{2 \pi \alpha_{\mathrm{em}}^{2}}{\beta Q^{4}} Y_{+} \sigma_{\mathrm{r}}^{\mathrm{D}(3)}
$$

where $Y_{+}=1+(1-y)^{2}$ and the reduced cross sections can be expressed in terms of two diffractive structure functions $F_{2}^{\mathrm{D}}$ and $F_{\mathrm{L}}^{\mathrm{D}}$. In the one-photon approximation, the relations are

$$
\begin{aligned}
& \sigma_{\mathrm{r}}^{\mathrm{D}(3)}=F_{2}^{\mathrm{D}(3)}\left(\beta, \xi, Q^{2}\right)-\frac{y^{2}}{Y_{+}} F_{\mathrm{L}}^{\mathrm{D}(3)}\left(\beta, \xi, Q^{2}\right), \\
& \sigma_{\mathrm{r}}^{\mathrm{D}(4)}=F_{2}^{\mathrm{D}(4)}\left(\beta, \xi, Q^{2}, t\right)-\frac{y^{2}}{Y_{+}} F_{\mathrm{L}}^{\mathrm{D}(4)}\left(\beta, \xi, Q^{2}, t\right) .
\end{aligned}
$$

Note that the structure functions $F_{2, \mathrm{~L}}^{\mathrm{D}(4)}$ have dimension giga-electron-volts to the negative second power, while 
$F_{2, \mathrm{~L}}^{\mathrm{D}(3)}$ are dimensionless. In this analysis, we neglect $Z^{0}$ exchange, though it should be included in future studies.

The reduced cross sections $\sigma_{\mathrm{r}}^{\mathrm{D}}$ depend on center-of-mass energy via $y=\frac{Q^{2}}{\xi \beta s}$. The $Y_{+}$factors ensure that in the region where $y$ is not too close to unity

$$
\sigma_{\mathrm{r}}^{\mathrm{D}} \simeq F_{2}^{\mathrm{D}}
$$

to good approximation.

Both $\sigma_{\mathrm{r}}^{\mathrm{D}(3)}$ and $\sigma_{\mathrm{r}}^{\mathrm{D}(4)}$ have been measured at the HERA collider [1,2,25-31] and used to obtain QCD-inspired parametrizations.

The standard perturbative QCD approach to diffractive cross sections is based on collinear factorization [5-7]. It was demonstrated that, similarly to the inclusive DIS cross section, the diffractive cross section can be written, up to terms of order $\mathcal{O}\left(1 / Q^{2}\right)$, in a factorized form

$$
\begin{aligned}
& d \sigma^{e p \rightarrow e X Y}\left(\beta, \xi, Q^{2}, t\right) \\
& =\sum_{i} \int_{\beta}^{1} d z d \hat{\sigma}^{e i}\left(\frac{\beta}{z}, Q^{2}\right) f_{i}^{\mathrm{D}}\left(z, \xi, Q^{2}, t\right),
\end{aligned}
$$

where the sum is performed over all parton flavors (gluon, $d$-quark, $u$-quark, etc.). The hard scattering partonic cross section $d \hat{\sigma}^{e i}$ can be computed perturbatively in QCD and is the same as in the inclusive deep inelastic scattering case. The long distance part $f_{i}^{\mathrm{D}}$ corresponds to the diffractive parton distribution functions, which can be interpreted as conditional probabilities for partons in the proton, provided the proton is scattered into the final state system $Y$ with specified 4-momentum $p^{\prime}$. They are evolved using the DGLAP evolution equations [32-35] similarly to the inclusive case. The analogous formula for the $t$-integrated structure functions reads

$F_{2 / \mathrm{L}}^{\mathrm{D}(3)}\left(\beta, \xi, Q^{2}\right)=\sum_{i} \int_{\beta}^{1} \frac{d z}{z} C_{2 / \mathrm{L}, i}\left(\frac{\beta}{z}\right) f_{i}^{\mathrm{D}(3)}\left(z, \xi, Q^{2}\right)$,

where the coefficient functions $C_{2 / \mathrm{L}, i}$ are the same as in inclusive DIS.

\section{SIMULATIONS FOR THE ELECTRON-PROTON DIS}

\section{A. Diffractive PDF parametrizations and HERA data}

Fits to the diffractive structure functions were performed by H1 [25] and ZEUS [29]. They both parametrize the diffractive PDFs in a two-component model, which is a sum of two exchange contributions, $\mathbb{P}$ and $\mathbb{R}$ :

$f_{i}^{\mathrm{D}(4)}\left(z, \xi, Q^{2}, t\right)=f_{\mathbb{P}}^{p}(\xi, t) f_{i}^{\mathbb{P}}\left(z, Q^{2}\right)+f_{\mathbb{R}}^{p}(\xi, t) f_{i}^{\mathbb{R}}\left(z, Q^{2}\right)$.
For both of these terms, proton vertex factorization is assumed, meaning that the diffractive exchange can be interpreted as colorless objects called a "Pomeron" or a "Reggeon" with parton distributions $f_{i}^{\mathbb{P}, \mathbb{R}}\left(\beta, Q^{2}\right)$. The flux factors $f_{\mathbb{P}, \mathbb{R}}^{p}(\xi, t)$ represent the probability that a Pomeron/ Reggeon with given values $\xi, t$ couples to the proton. They are parametrized using the form motivated by Regge theory,

$$
f_{\mathbb{P}, \mathbb{R}}^{p}(\xi, t)=A_{\mathbb{P}, \mathbb{R}} \frac{e^{B_{\mathbb{P}, \mathbb{R}} t}}{\xi^{2 \alpha_{\mathbb{P}, \mathbb{R}}(t)-1}},
$$

with a linear trajectory $\alpha_{\mathbb{P}, \mathbb{R}}(t)=\alpha_{\mathbb{P}, \mathbb{R}}(0)+\alpha_{\mathbb{P}, \mathbb{R}}^{\prime} t$. The diffractive PDFs relevant to the $t$-integrated cross sections read

$f_{i}^{\mathrm{D}(3)}\left(z, \xi, Q^{2}\right)=\phi_{\mathbb{P}}^{p}(\xi) f_{i}^{\mathbb{P}}\left(z, Q^{2}\right)+\phi_{\mathbb{R}}^{p}(\xi) f_{i}^{\mathbb{R}}\left(z, Q^{2}\right)$,

with

$$
\phi_{\mathbb{P}, \mathbb{R}}^{p}(\xi)=\int d t f_{\mathbb{P}, \mathbb{R}}^{p}(\xi, t) .
$$

Note that the notions of Pomeron and Reggeon used here to model hard diffraction in DIS are, in principle, different from those describing the soft hadron-hadron interactions; in particular, the parameters of the fluxes may be different.

The diffractive parton distributions of the Pomeron at the initial scale $\mu_{0}^{2}=1.8 \mathrm{GeV}^{2}$ are parametrized as

$$
z f_{i}^{\mathbb{P}}\left(z, \mu_{0}^{2}\right)=A_{i} z^{B_{i}}(1-z)^{C_{i}},
$$

where $i$ is a gluon or a light quark. In the diffractive parametrizations, all the light quarks (antiquarks) are assumed to be equal. For the treatment of heavy flavors, a variable flavor number scheme (VFNS), in which the charm and bottom-quark DPDFs are generated radiatively via DGLAP evolution and no intrinsic heavy-quark distributions are assumed, is adopted. The structure functions are calculated in a general-mass variable flavor number scheme (GM-VFNS) [36,37], which ensures a smooth transition of $F_{2, \mathrm{~L}}$ across the flavor thresholds by including $\mathcal{O}\left(m_{h}^{2} / Q^{2}\right)$ corrections. The parton distributions for the Reggeon component are taken from a parametrization which was obtained from fits to the pion structure function [38,39].

In Eq. (9), the normalization factors of fluxes, $A_{\mathbb{P}, \mathbb{R}}$ and of DPDFs, $A_{i}$ enter in the product. To resolve the ambiguity, we fix ${ }^{1} A_{\mathbb{P}}$ and use $f_{i}^{\mathbb{R}}\left(z, Q^{2}\right)$ normalized to

\footnotetext{
${ }^{1}$ Here, as in the HERA fits, $A_{\mathbb{P}}$ is fixed by normalizing $\phi_{\mathbb{P}}^{p}(0.003)=1$.
} 

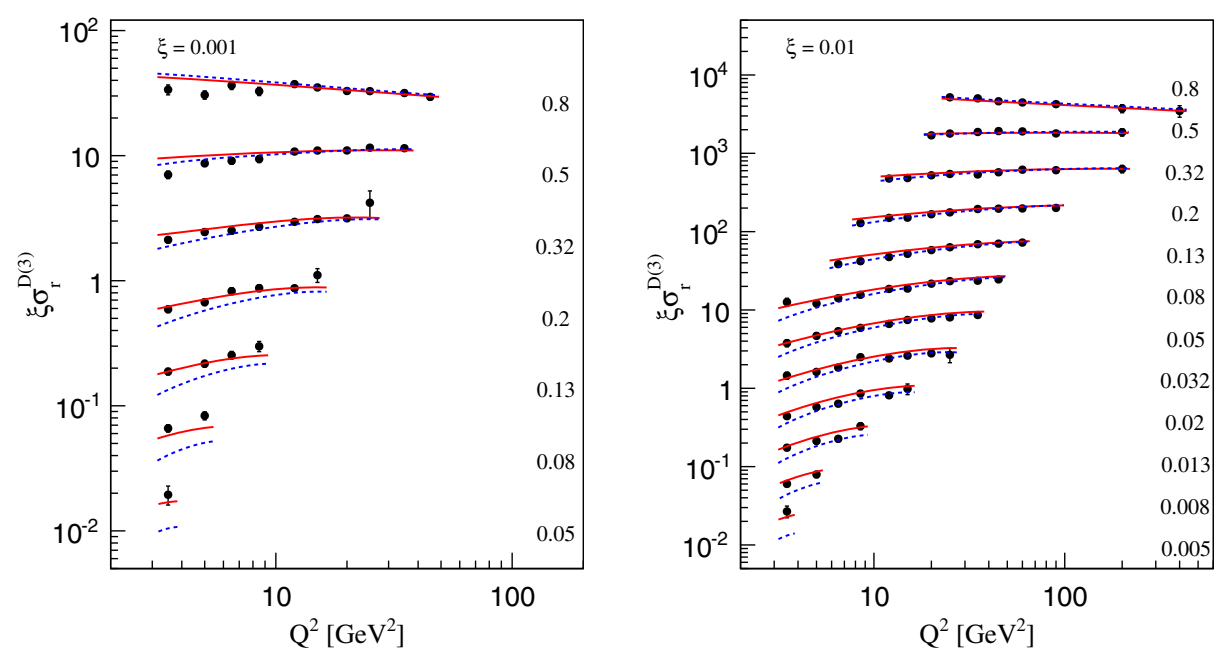

FIG. 2. Experimental data from the H1 Collaboration at HERA [31] on the reduced diffractive cross section as a function of $Q^{2}$ in bins of $\beta$ for two values of $\xi=0.001$ (left) and $\xi=0.01$ (right). The lines indicate predictions from two fits to older data: H1 2006 fit B (dotted, blue) and ZEUS-SJ (solid, red). The values shown are scaled by $3^{k}$ for $k=0,1, \ldots$ upward.

the pion structure function, which results in $A_{i}$ and $A_{\mathbb{R}}$ being well-defined free fit parameters.

There are different types of diffractive fits available in the literature. Here, we mention the next-to-leading-order (NLO) parametrizations from HERA relevant to the current study:

(i) Fit $S$ : All parameters $A_{i}, B_{i}, C_{i}$ are free, as are $A_{\mathbb{R}}$ and $\alpha_{\mathbb{P}, \mathbb{R}}(0)$ (nine parameters). This is the ZEUS-S fit.

(ii) Fit $C$ : Parameters $B_{g}, C_{g}$ are set to zero, resulting in a constant gluon density at the starting scale for $\mathrm{QCD}$ evolution. This corresponds to the $\mathrm{H} 1 \mathrm{fit} \mathrm{B}$ fit when $A_{\mathbb{R}}$ and $\alpha_{\mathbb{P}}(0)$ are free (six parameters) and to the ZEUS-C fit when $A_{\mathbb{R}}$ and $\alpha_{\mathbb{P}, \mathbb{R}}(0)$ are free (seven parameters).

(iii) Fit SJ: All parameters $A_{i}, B_{i}, C_{i}$ are free. In addition, dijet production data are used to constrain the gluon. This amounts to the ZEUS-SJ fit when $A_{\mathbb{R}}$ and $\alpha_{\mathbb{P}, \mathbb{R}}(0)$ are free (nine parameters) and to the H12007 fit [40] when $A_{\mathbb{R}}$ and $\alpha_{\mathbb{P}}(0)$ are free (eight parameters).

Note that fit $\mathrm{S}$ and fit SJ share the same functional form, differing only in the use of dijet data in the latter. In the current work, the ZEUS-SJ fit predictions are used for pseudodata simulation, and the fitting analysis is performed with the fit-S parametrization model, i.e., using the same parametrization.

In Fig. 2, we show some example of recent HERA data [31] compared with two fits, H1 fit B and ZEUS-SJ. Note that the fits were performed to data older than shown in the figure.

\section{B. LHeC and FCC-eh kinematics compared with HERA data}

The kinematic range in $\left(\beta, Q^{2}, \xi\right)$ is restricted by the following cuts:

(i) $Q^{2} \geq 1.8 \mathrm{GeV}^{2}$ : due to the fact that the initial distribution for the DGLAP evolution is parametrized at $\mu_{0}^{2}=1.8 \mathrm{GeV}^{2}$. The renormalization and factorization scales are taken to be equal to $Q^{2}$.

(ii) $\xi<0.4$ : by physical and experimental limitations. This rather high $\xi$ value is an experimental challenge and physically enters the phase-space region where the Pomeron contribution should become negligible. Within the two-component model of Eq. (9), at high $\xi$, the cross section is dominated by the secondary Reggeon contribution, which is poorly fixed by the HERA data. We present this high- $\xi$ (greater than 0.1) region for illustrative purposes and for the sake of discussion of the fit results in Sec. IV.

In Fig. 3, the accessible kinematic range in $\left(x, Q^{2}\right)$ is shown for four machines: HERA, LHeC, FCC-eh and EIC. For the $\mathrm{LHeC}$ design, the range in $x$ is increased by a factor approximately 20 over HERA and the maximum available $Q^{2}$ by a factor approximately 100 . The FCC-eh machine would further increase this range with respect to $\mathrm{LHeC}$ by roughly one order of magnitude in both $x$ and $Q^{2}$. We also show the EIC kinematic region for comparison.

In Figs. 4 and 5, the phase space in $\left(\beta, Q^{2}\right)$ is shown for fixed $\xi$ for the LHeC and FCC-eh, respectively. Both machines probe very small values of $\xi$, the $\mathrm{LHeC}$ reaching $10^{-4}$ with a wide range of $\beta$ and the FCC-eh extending $\xi$ down to $10^{-5}$. Of course, the range in $\beta$ and $\xi$ is correlated since $x=\beta \xi$. Therefore, for small values of $\xi$, only large values of $\beta$ are accessible, while for large $\xi$, the range in $\beta$ extends to very small values. Above the solid, horizontal line labeled $m_{t}^{2}$, the top-quark DPDF comes into play, and above the dashed line, the $t \bar{t}$ production channel opens.

\section{Pseudodata for diffractive structure functions}

The reduced cross sections are extrapolated using Eqs. (5) and (8) with the ZEUS-SJ DPDFs. Following 


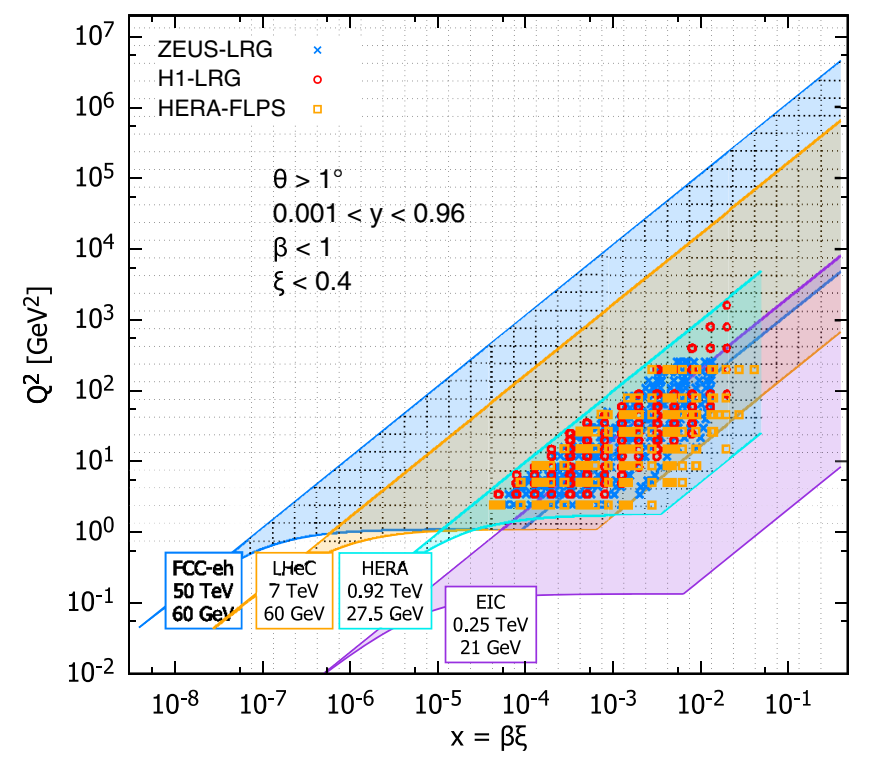

FIG. 3. Kinematic phase space for inclusive diffraction in $\left(x, Q^{2}\right)$ for the EIC (magenta region), the LHeC (orange region), and the FCC-eh (dark blue region) as compared with the HERA data (light blue region, ZEUS-LRG [26], H1-LRG [31], and HERA-FLPS [41]). The acceptance limit for the electron in the detector design has been assumed to be $1^{\circ}$, and we take $\xi<0.4$.

the scenario of the ZEUS fit [29], we work within the VFNS scheme at NLO accuracy. The transition scales for DGLAP evolution are fixed by the heavy-quark masses, $\mu^{2}=m_{h}^{2}$, and the structure functions are calculated in the Thorne-Roberts GM-VFNS [42]. The Reggeon PDFs are taken from the Gluck-Reya-Vogt (GRV) pion set [39]; the numerical parameters are taken from Tables 1 and 3 of Ref. [29]; and heavy-quark masses are $m_{c}=1.35 \mathrm{GeV}, m_{b}=4.3 \mathrm{GeV}$, and $\alpha_{\mathrm{s}}\left(M_{Z}^{2}\right)=0.118$.

The model has a non-negligible Reggeon contribution, which is hard to constrain from HERA data. It increases with increasing $\xi$ and gives a substantial contribution in the region $\xi>0.01$ for both the $\mathrm{LHeC}$ and the FCC-eh kinematics. Thus, it is a source of a large uncertainty on the predictions in this region.

The HERA kinematics give no access to the top-quark region, and thus the model provides no reliable contributions from the top quarks. In the following simulations, the top-quark contribution to the cross section is neglected, so the extrapolated cross sections are likely underestimated for $Q^{2}>m_{t}^{2}$ and $M_{X}>2 m_{t}$-the significance of the top region is discussed in Sec. IV.

The pseudodata were generated using the extrapolation of the fit to HERA data, which provides the central values, amended with a random Gaussian smearing with standard deviation corresponding to the relative error $\delta$. An uncorrelated $5 \%$ systematic error was assumed giving a total error

$$
\delta=\sqrt{\delta_{\text {sys }}^{2}+\delta_{\text {stat }}^{2}}
$$

The statistical error was computed assuming a very modest integrated luminosity of $2 \mathrm{fb}^{-1}$; see Refs. [20,21]. For the binning adopted in this study, the statistical uncertainties have a very small effect on the uncertainties in the extracted DPDFs. Obviously, a much larger luminosity would allow a denser binning that would result in smaller DPDF uncertainties.

Note that our aim is not to provide a rigorous prediction of the reduced cross section or its full uncertainty. Such a
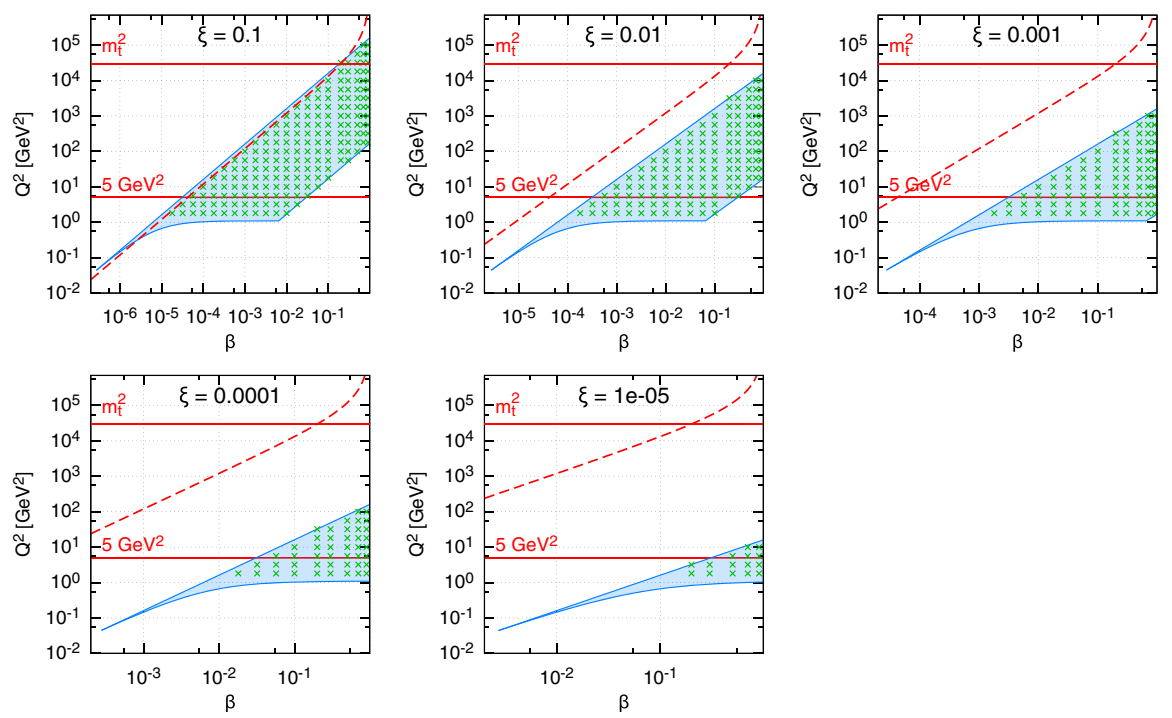

FIG. 4. Kinematic phase space for inclusive diffraction in $\left(\beta, Q^{2}\right)$ for fixed values of $\xi$ for the LHeC design. The horizontal lines indicate, correspondingly, $Q^{2}=5 \mathrm{GeV}^{2}$, the lowest data value for the DGLAP fit performed in this study, and $m_{\mathrm{t}}^{2}$, the six-flavor threshold. The dashed line marks the kinematic limit for $t \bar{t}$ production. 

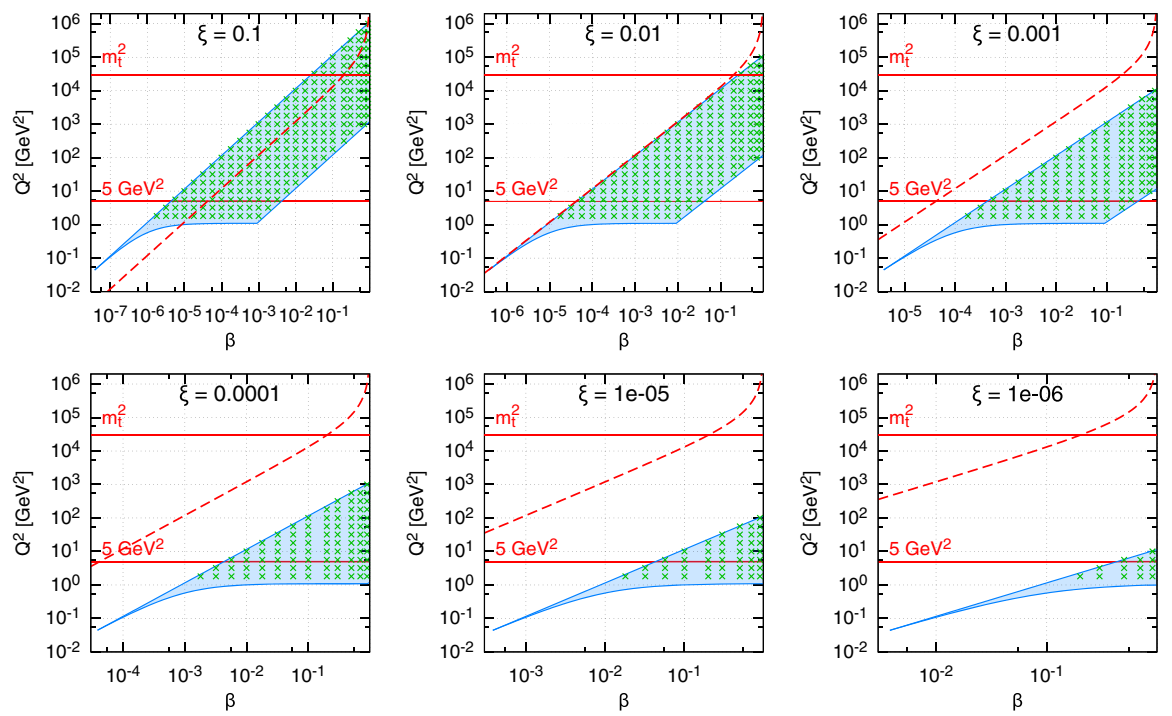

FIG. 5. Kinematic phase space for inclusive diffraction in $\left(\beta, Q^{2}\right)$ for fixed values of $\xi$ for the FCC-eh design. The horizontal lines indicate, correspondingly, $Q^{2}=5 \mathrm{GeV}^{2}$, the lowest data value for the DGLAP fit performed in this study, and $m_{\mathrm{t}}^{2}$, the six-flavor threshold. The dashed line marks the kinematic limit for $t \bar{t}$ production.

study would need to take account of theory uncertainties such as those stemming from the order in the perturbative expansion, the functional form of the initial conditions for DGLAP evolution, and the values of the strong coupling constant and heavy-quark masses. Our goal is simply to establish the extent to which data from new machines can reduce the existing experimental uncertainties in DPDFs relative to those from the fits to HERA data.

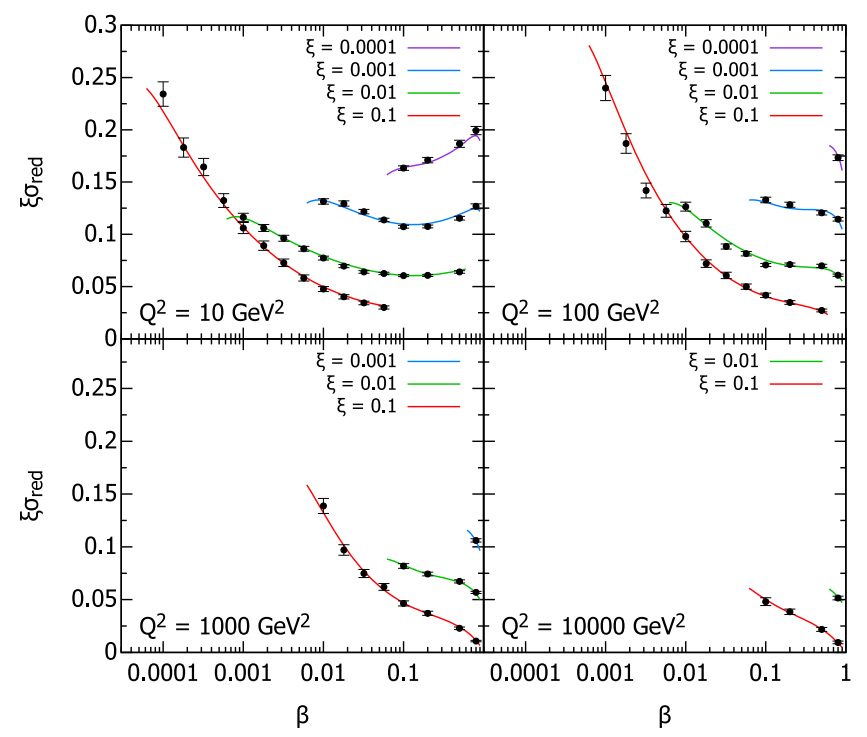

FIG. 6. Selected subset of the simulated data for the diffractive reduced cross section as a function of $\beta$ in bins of $\xi$ and $Q^{2}$ for $e p$ collisions at the LHeC. The curves for $\xi=0.01,0.001,0.0001$ are shifted up by $0.04,0.08,0.12$, respectively.
In Figs. 6 and 7, we show a subset of the simulated data for the diffractive reduced cross section $\xi \sigma_{\text {red }}$ as a function of $\beta$ in selected bins of $\xi$ and $Q^{2}$ for the LHeC and FCC-eh cases, respectively. For the most part, the errors are very small and are dominated by the systematics. The breaking of Regge factorization evident at large $\xi$ comes from the large Reggeon contribution in that region, the validity of which could be further investigated at the $\mathrm{LHeC}$ and FCC-eh.

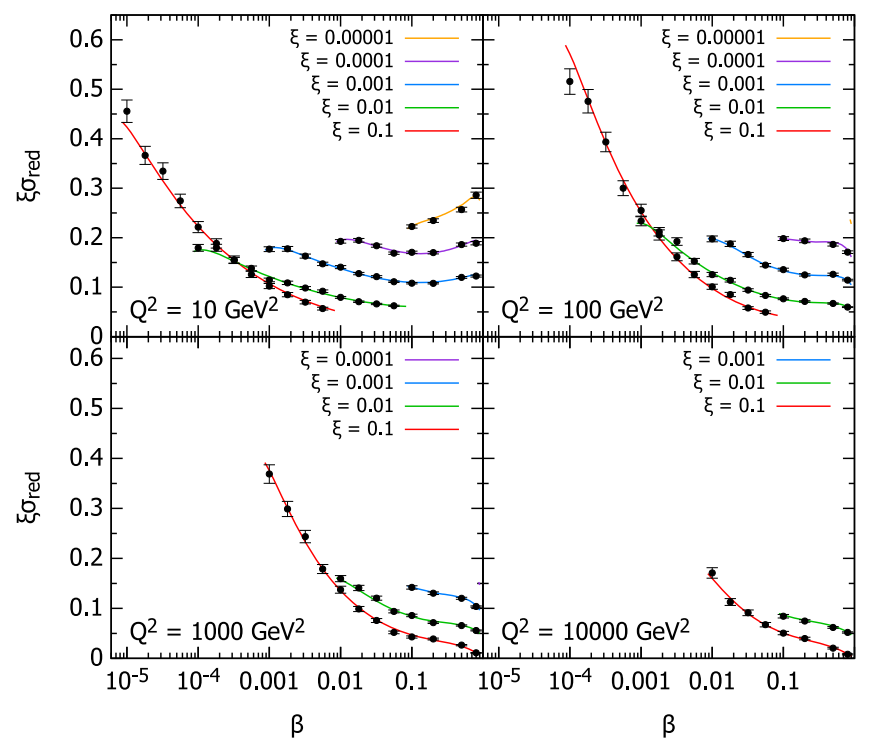

FIG. 7. Selected subset of the simulated data for the diffractive reduced cross section as a function of $\beta$ in bins of $\xi$ and $Q^{2}$ for $e p$ collisions at the FCC-eh. The curves for $\xi=0.01,0.001,0.0001$, 0.00001 are shifted up by $0.04,0.08,0.12,0.16$, respectively. 


\section{POTENTIAL FOR CONSTRAINING DIFFRACTIVE PDFs AT THE LHeC AND FCC-eh}

\section{A. Fitting methodology and results}

With the aim of establishing the experimental precision with which DPDFs could be extracted when LHeC and FCC-eh data become available, we generate the central values of the pseudodata using the central set of the ZEUSSJ fit that are distributed according to a Gaussian with experimental width, Eq. (14), that also provides the uncertainty in the pseudodata. We then include the pseudodata in a fit using the same functional form and, as expected, obtain a $\chi^{2} /$ ndf $\sim 1$, which demonstrates the consistency of the approach. ${ }^{2}$ The fact that the $\chi^{2}$ is fully acceptable suggests that using a more flexible form for each parton species, or adding more species by allowing parton decomposition, cannot improve the fit to the pseudodata in a meaningful way. Obviously, it may turn out when real data become available that the functional form used in our work is not able to produce a satisfactory fit and improvements of the parametrization would then be required, ideally with an assessment of the associated uncertainties. Understanding and quantifying such parametrization biases and uncertainties is a most important subject in its own right; see the comments at the end of Sec. IV B., which go beyond the limited scope of this work.

To evaluate the precision with which the DPDFs can be determined, several pseudodata sets, corresponding to independent random error samples, were generated. Each pseudodata set was fitted to the reduced cross sections defined by Eqs. (5a) and (8) in the DPDF model of Sec. III A.

The minimal value of $Q^{2}$ for the data considered in the fits was set to $Q_{\min }^{2}=5 \mathrm{GeV}^{2}$. The reason for this cutoff is to show the feasibility of the fits including just the range in which the standard twist-2 DGLAP evolution is expected to be trustable. At HERA, the $Q_{\min }^{2}$ values giving acceptable DGLAP (twist-2) fits were 8 [25] and $5 \mathrm{GeV}^{2}$ [26] for $\mathrm{H} 1$ and ZEUS, respectively. It is expected that if there are any higher-twist effects, for example due to parton saturation, they should become visible in the lower- $Q^{2}$ region. DGLAP fits to the diffractive data are known to not describe the data very well in this region, which may point to the importance of the higher-order or higher-twist corrections.

It is possible that a more flexible functional form would eventually be able to fit such data from the new machines without resorting to dynamics beyond twist-2 DGLAP, but with the amount and precision of HERA data, no evidence

\footnotetext{
${ }^{2}$ As a cross-check of the method, we have performed a fit to the data simulated in the HERA kinematic region with HERA-like experimental errors approximately $10 \%$, and we recovered the ZEUS-SJ fit results and uncertainties with very good accuracy. We have also modified the experimental uncertainties by a factor 2 and observed that the uncertainties in the extracted DPDFs are changed by the same factor.
}

for this was found. Note that phenomenological studies which include higher-twist corrections indeed describe the HERA data in this region better than the pure DGLAP evolution [43].

The maximum value of $\xi$ was set by default to $\xi_{\max }=0.1$, above which the cross section starts to be dominated by the Reggeon exchange. The effects of relaxing both limits $Q_{\min }^{2}$ and $\xi_{\max }$ are described below. The region above the top threshold was not considered in the fits. This point, however, should be addressed in future studies; the top contribution has a negligible impact for the LHeC but some impact for the FCC-eh.

The binning adopted in this study corresponds roughly to four bins per order of magnitude in each of $\xi, \beta, Q^{2}$. For $Q_{\text {min }}^{2}=5 \mathrm{GeV}^{2}, \xi_{\max }=0.1$, and below the top threshold, this results in 1229 and 1735 pseudodata points for the LHeC and FCC-eh, respectively. The top-quark region adds 17 points for the $\mathrm{LHeC}$ and 255 for FCC-eh. Lowering $Q_{\text {min }}^{2}$ down to $1.8 \mathrm{GeV}^{2}$, we get 1589 and 2171 pseudodata points, while increasing $\xi$ up to 0.32 adds around 180 points for both machines.

The potential for the determination of the gluon DPDF was investigated by fitting the inclusive diffractive DIS pseudodata with two models, $\mathrm{S}$ and $\mathrm{C}$ of Sec. III A with $\alpha_{\mathbb{P}, \mathbb{R}}(0)$ fixed, in order to focus on the shape of the Pomeron's PDFs. At HERA, both $\mathrm{S}$ and $\mathrm{C}$ fits provide equally good descriptions of the data with $\chi^{2} /$ ndf $=1.19$ and 1.18, respectively, despite different gluon DPDF shapes. The LHeC pseudodata are much more sensitive to gluons, resulting in $\chi^{2} /$ ndf values of 1.05 and 1.4 for the $\mathrm{S}$ and $\mathrm{C}$ fits, respectively. This motivates the use of the larger number of parameters in the fit-S model, which we employ in the further studies. It also shows clearly the potential of the LHeC and the FCC-eh to better constrain the low- $x$ gluon and, therefore, unravel eventual departures from standard linear evolution.

\section{B. DPDFs uncertainties}

In Figs. 8 and 9, the diffractive gluon and quark distributions are shown for the LHeC and FCC-eh, respectively, as a function of $z$ for fixed scales $\mu^{2}=6,20,60,200 \mathrm{GeV}^{2}$. The bands labeled A, B, and C denote fits to three statistically independent pseudodata replicas, obtained from the same central values and statistic and systematic uncertainties. Hereafter, the bands shown correspond to $\Delta \chi^{2}=2.7$ uncertainty (90\% C.L.). Also, the extrapolated ZEUS-SJ DPDFs are shown with error bands marked by the / hatched area. Note that the depicted uncertainty bands come solely from experimental errors, neglecting theoretical sources, such as fixed input parameters and parametrization biases. The extrapolation beyond the reach of $\mathrm{LHeC} / \mathrm{FCC}$-eh is marked in gray, and the HERA kinematic limit is marked with the vertical dotted line. The stability of the results with respect to the replica used for the analysis is evident, so in the following, 

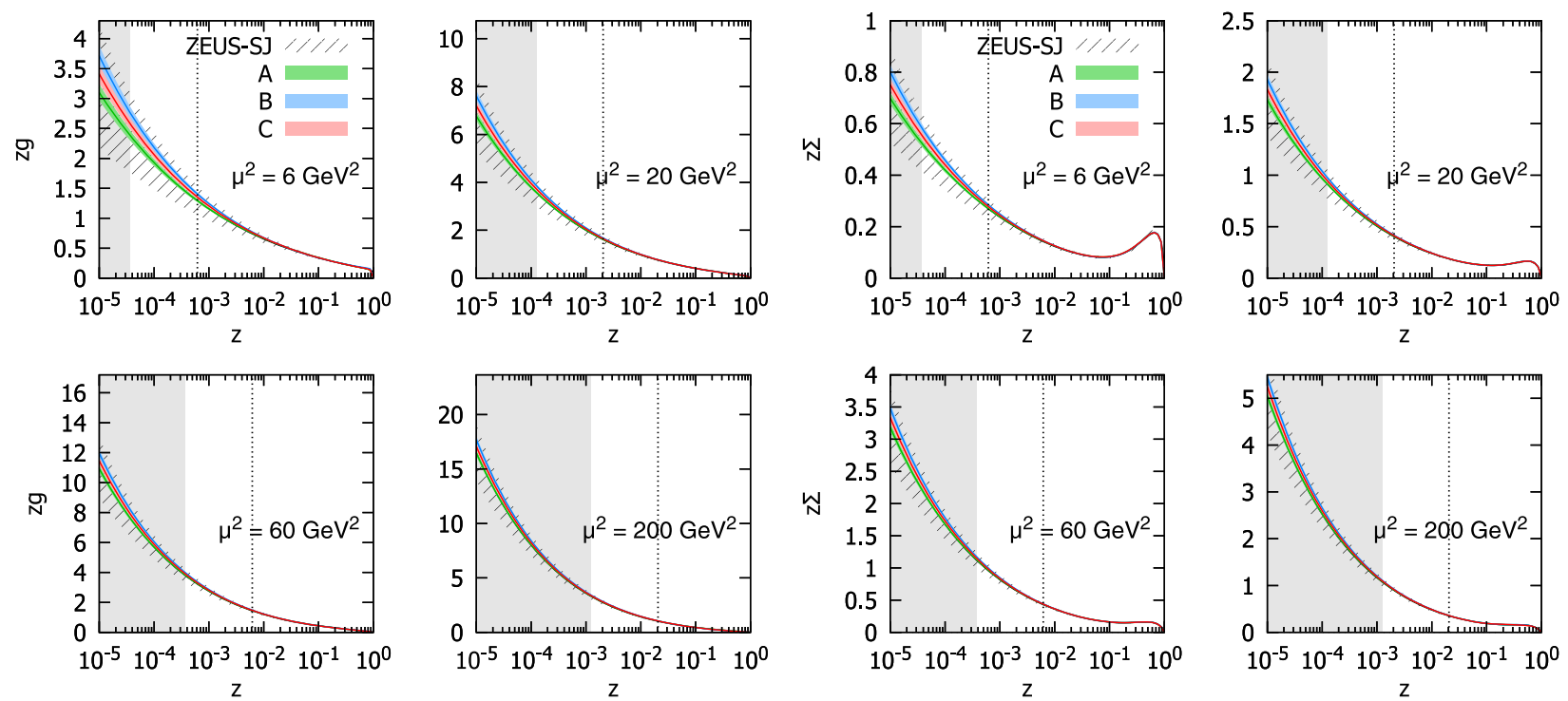

FIG. 8. Diffractive PDFs for gluon and quark in the LHeC kinematics as a function of momentum fraction $z$ for fixed values of scale $\mu^{2}$. Results of fits to three (A, B, and C) pseudodata replicas are shown together with the experimental error bands. For comparison, the extrapolated ZEUS-SJ fit is also shown (black) with error bands marked with the hatched pattern. The vertical dotted lines indicate the HERA kinematic limit. The bands indicate only the experimental uncertainties; see the text.
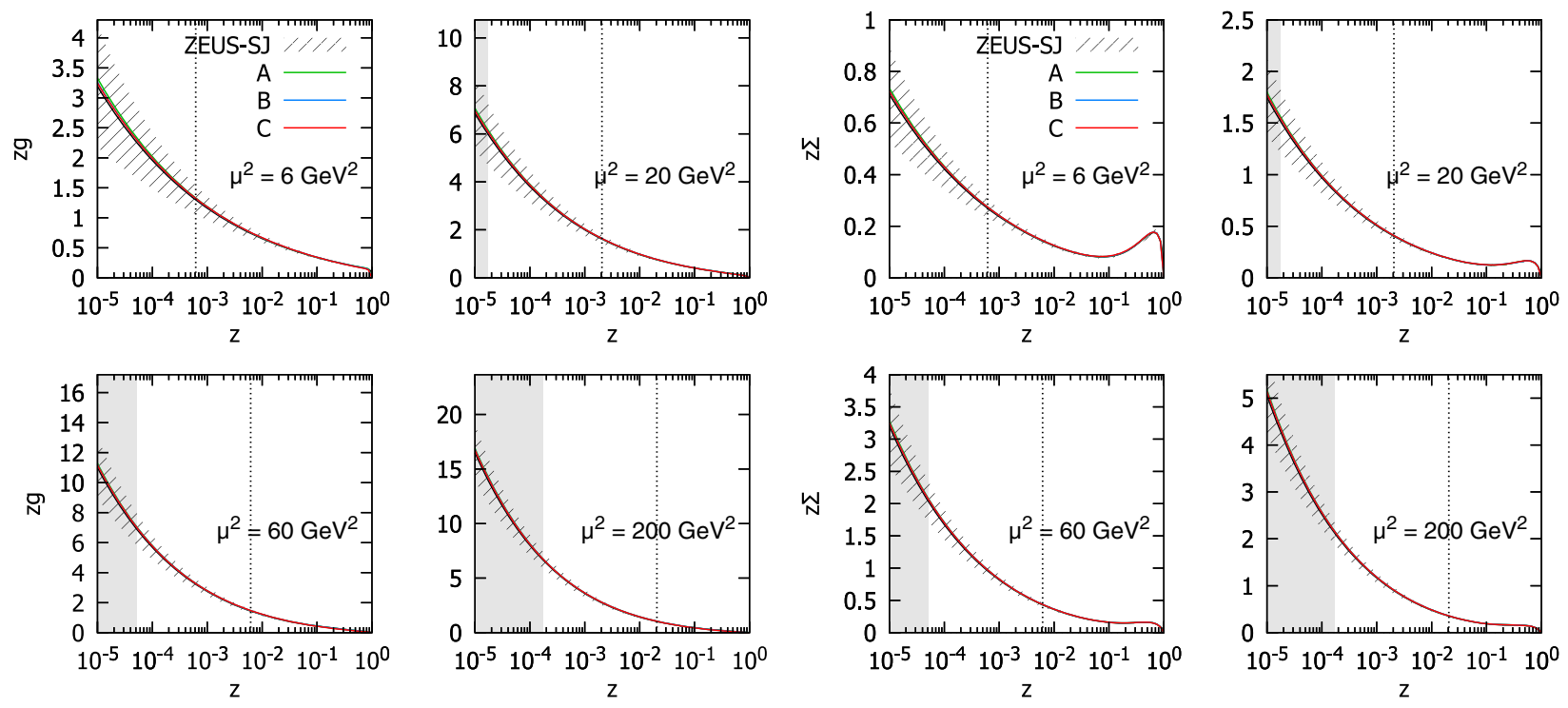

FIG. 9. Identical to Fig. 8, but in the FCC-eh kinematics. The bands indicate only the experimental uncertainties; see the text.

only one will be employed. The DPDFs determination accuracy improves with respect to HERA by a factor of 5-7 for the LHeC and 10-15 for the FCC-eh.

For a better illustration of the precision, in Figs. 10-12, the relative uncertainties are shown for parton distributions at different scales. In Fig. 10, the upper plots correspond to the $\mathrm{LHeC}$, and the lower ones correspond to the FCC-eh scenarios. The different bands show the variation with the upper cut on the available $\xi$ range, from 0.01 to 0.32 . We observe only a modest improvement in the achievable accuracy of the extracted DPDFs with the change of $\xi$ by an order of magnitude from 0.01 to 0.1 . An almost negligible effect is observed when further extending the $\xi$ range up to 0.32 . This is encouraging, since the measurement for the very large values of $\xi$ is challenging. It reflects the dominance of the secondary Reggeon in this region.

In Fig. 11, we show the variation of the relative precision with the change of the minimal value of $Q^{2}$ from $1.8 \mathrm{GeV}^{2}$ (curves) to $5 \mathrm{GeV}^{2}$ (bands). The $\mathrm{LHeC}$ scenario is indicated in green. and FCC-eh is in red. There is a quite substantial effect on the achieved precision depending on the minimal value of $Q^{2}$. This is not only related to the fact 

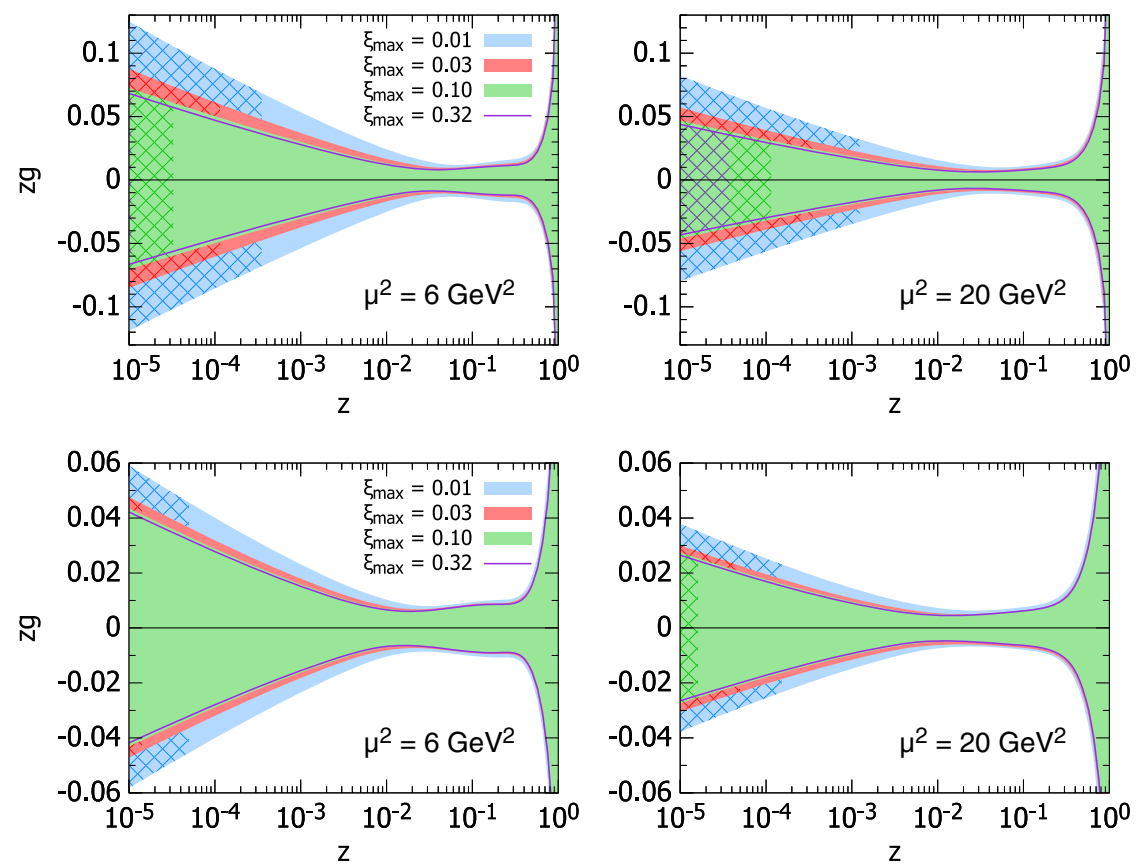

FIG. 10. Relative uncertainties on the diffractive gluon PDFs for the LHeC kinematics (upper panel) and FCC-eh kinematics (lower panel). Two different choices of scales are considered $\mu^{2}=6$ and $\mu^{2}=20 \mathrm{GeV}^{2}$. The blue, red, and green bands and magenta line correspond to different maximal values of $\xi=0.01,0.03,0.1,0.32$, respectively. The cross-hatched areas show kinematically excluded regions. The bands indicate only the experimental uncertainties; see the text.

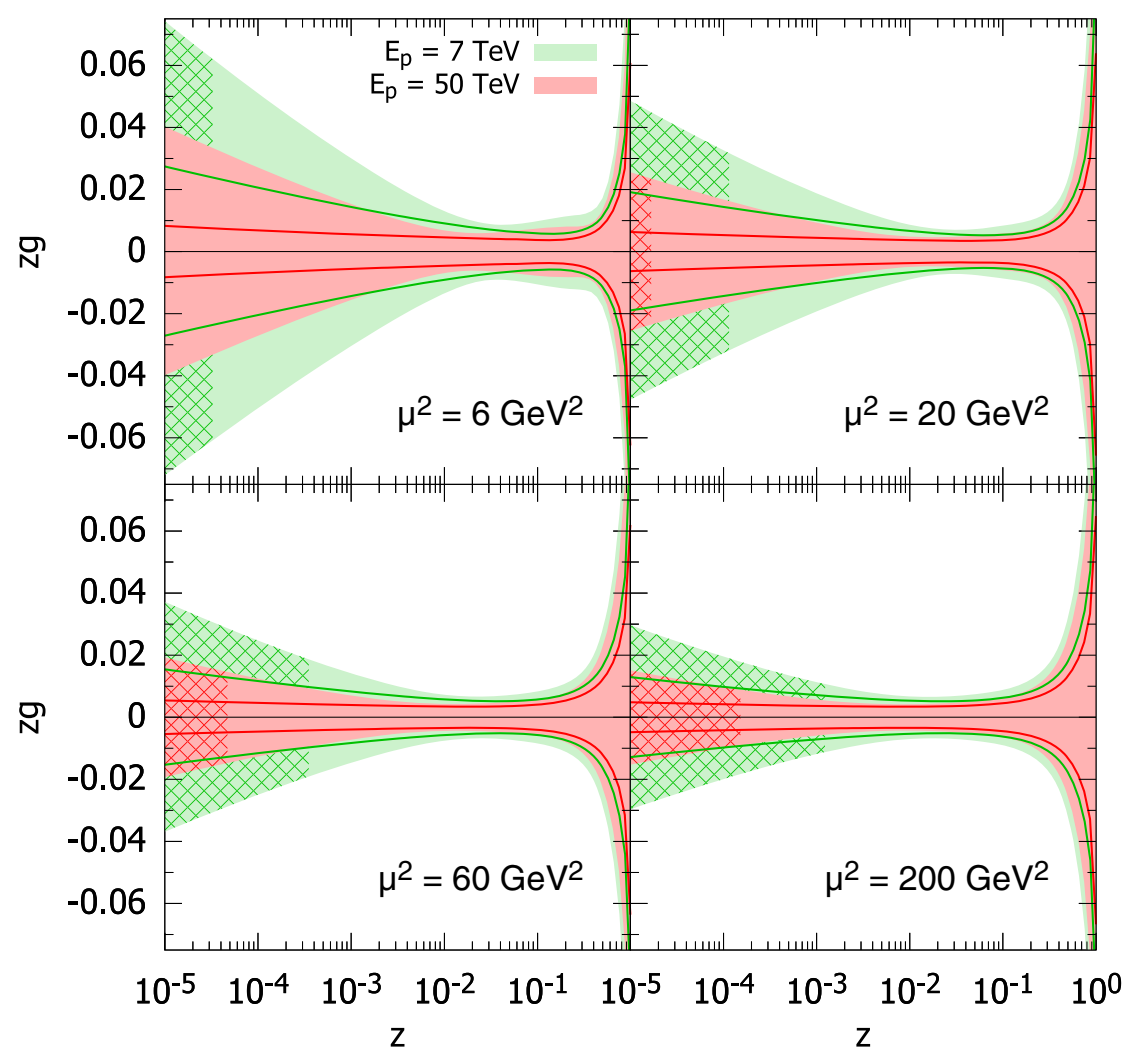

FIG. 11. Relative uncertainties on the diffractive gluon PDF extraction for four distinct scales $\mu^{2}=6,20,60,200 \mathrm{GeV}^{2}$. The bands correspond to the choice of the high cutoff on the data included in the fit $Q_{\min }^{2}=5 \mathrm{GeV}^{2}$, and the lines correspond to the lower choice $Q_{\min }^{2}=1.8 \mathrm{GeV}^{2}$. The green color corresponds to the LHeC scenario, and red corresponds to the FCC-eh scenario. The cross-hatched areas show kinematically excluded regions. The bands indicate only the experimental uncertainties; see the text. 

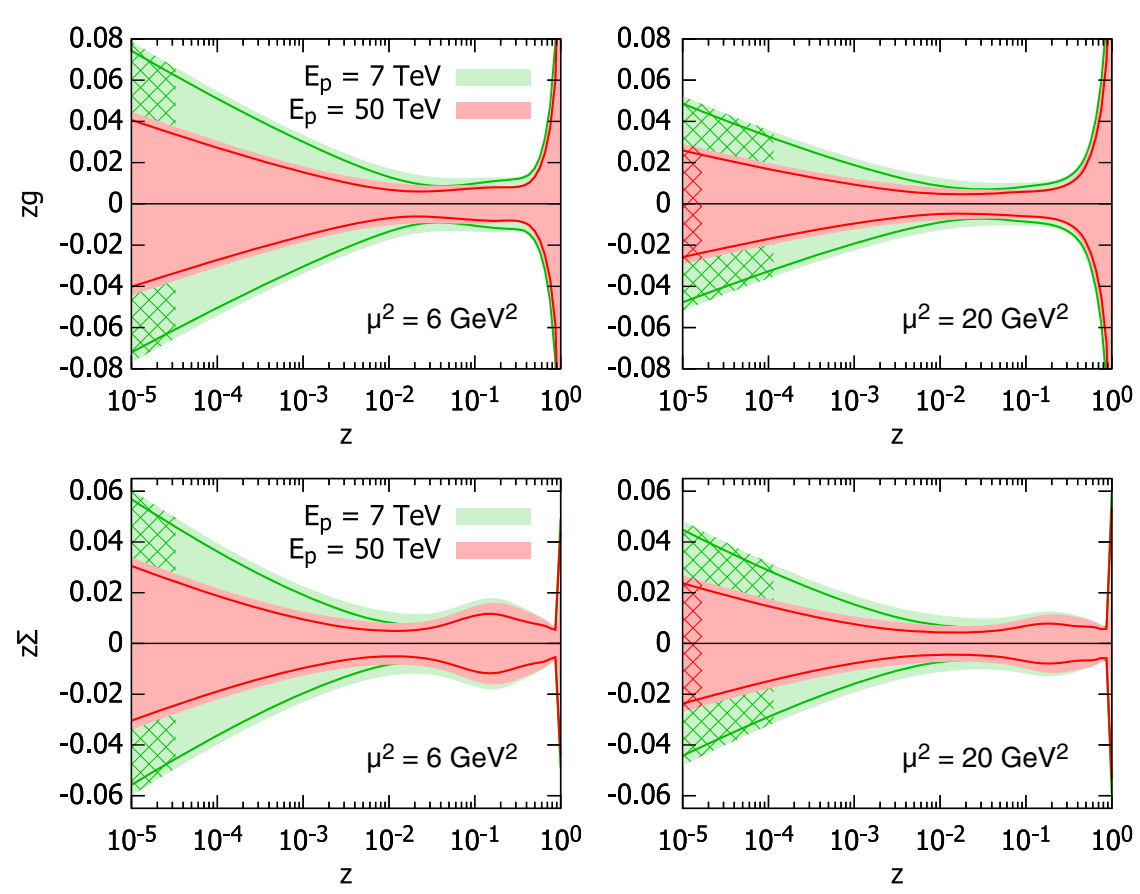

FIG. 12. Relative uncertainties on the diffractive PDFs for different numbers of free fit parameters, 7 and 9. Two different choices of scales are considered $\mu^{2}=6$ and $20 \mathrm{GeV}^{2}$. The green and red bands correspond to the nine-parameter fits for the LHeC and FCC-eh scenarios, respectively. The continuous lines delimit the seven-parameter fit uncertainty. The cross-hatched areas show kinematically excluded regions. The bands indicate only the experimental uncertainties; see the text.

that the number of pseudodata points is larger by about 300 in each case but is primarily due to the fact that acceptance across the full range of $z$ in this region is crucial for constraining the initial condition for the DGLAP evolution. The more data points are in the region closer to the starting distribution, the better it is constrained, particularly at low and medium values of $Q^{2}$ and $z$. Figure 11 also demonstrates that both machines will be very sensitive to this region and therefore potentially able to constrain higher twists and/or saturation effects.

In Fig. 12, we show the effect on the relative uncertainties for quarks and gluons of making $\alpha_{\mathbb{P}, \mathbb{R}}(0)$ free fit parameters. The increased number of fitting parameters from 7 to 9 has a very small effect on the DPDF uncertainties. In addition, we note that for low $x$ values the quark and gluon uncertainties are similar, with quark uncertainties being smaller by about $20 \%$. There is, however, a marked difference in the uncertainties for quarks and gluons at large values of $z$.

For a concluding comment in this section, let us briefly discuss the influence of the functional form of the initial conditions for DGLAP evolution on the uncertainties obtained from fitting pseudodata. For this purpose, the following exercise has been performed. We have included four additional parameters in (13) through multiplying it by $1+D_{i} z+E_{i} \sqrt{z}$, for $i$ equal to a gluon or a light quark. We have checked that such a form, with given values of $D_{i}, E_{i}$, is not in large disagreement with the HERA diffractive cross sections. This form and choice of parameters has been used to generate pseudodata in the HERA and LHeC kinematics. These pseudodata have first been fitted using our standard ZEUS-SJ form, $D_{i}=E_{i}=0$, and then including $D_{i}, E_{i}$ one by one as additional fitting parameters. We generically observe that, in both kinematic domains, the improvement in the $\chi^{2} /$ ndf when increasing the number of parameters is marginal-from one per hundred to less than one per thousand-and the size of the uncertainty bands is not larger than in the ZEUS-SJbased analysis. Therefore, in the kinematic ranges that we are exploring and with this functional form, we have been unable to quantify a meaningful parametrization uncertainty. Obviously, these results and conclusions are linked to a given functional form and approach to estimate the uncertainties. A different functional form or the use of, e.g., the neural network parton distribution functions (NNPDF) approach [44] instead of the Hessian method [45] may be essayed. But the answers obtained using these different forms and approaches would be, as in our case, linked to the specific choices and methodologies. We take these facts as indicative that a proper treatment of parametrization uncertainties can only be addressed on the basis of real data and supportive of our strategy here of focusing solely on the experimental uncertainties.

\section{DIFFRACTIVE DEEP INELASTIC SCATTERING OFF NUCLEI}

Electron-nucleus $(e \mathrm{~A})$ collisions are also possible at the $\mathrm{LHeC}$ and the FCC-eh with large integrated luminosities, 

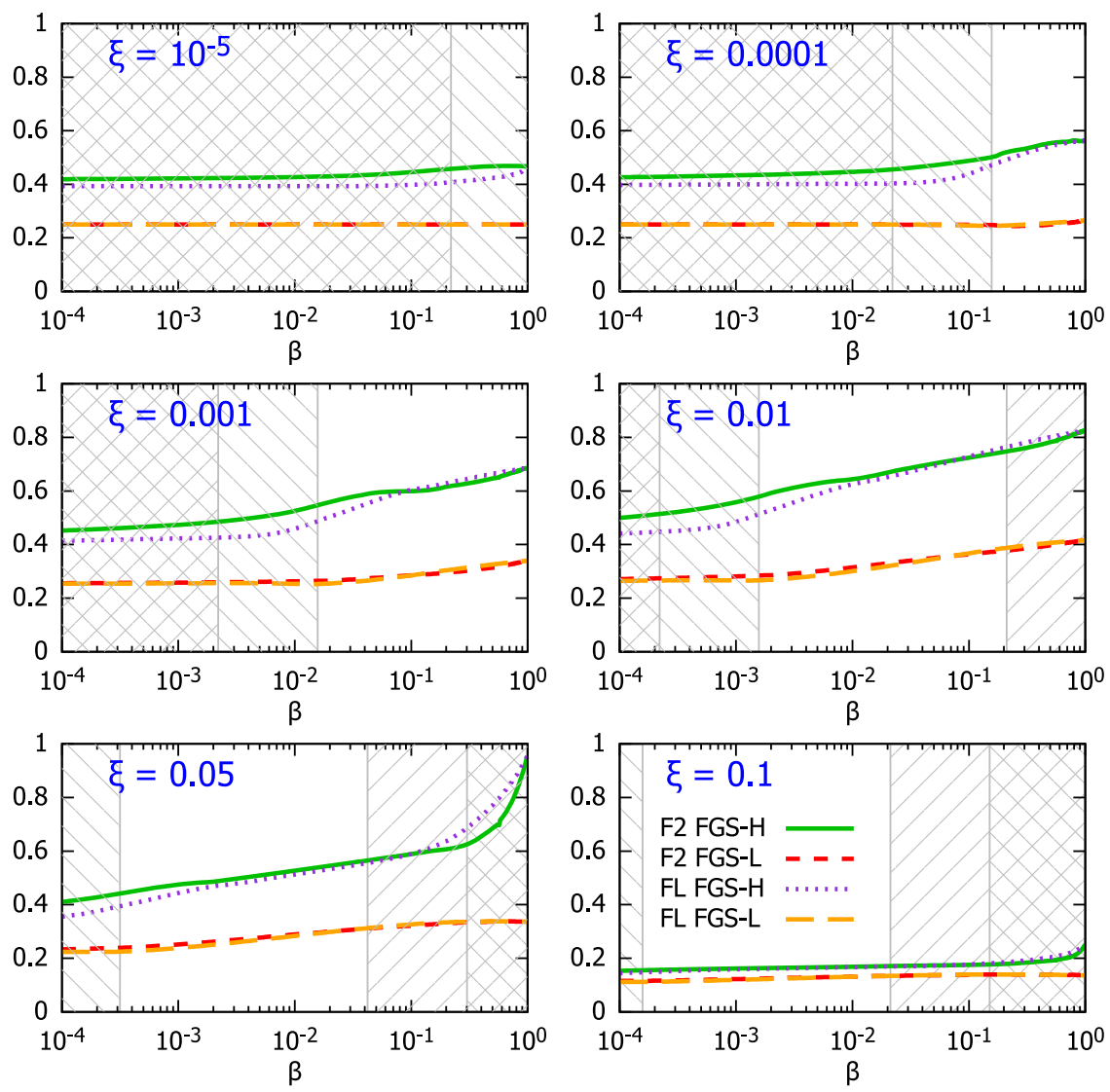

FIG. 13. Nuclear modification factor, Eq. (15), for $F_{2}^{D(3)}$ and $F_{L}^{D(3)}$ in ${ }^{208} \mathrm{~Pb}$ vs $\beta$, at $Q^{2}=10 \mathrm{GeV}^{2}$ and for different $\xi$, for the models $\mathrm{H}$ and L in Ref. [9]. The $\backslash$ and / hatched areas show kinematically excluded regions for $E=2.76$ and $19.7 \mathrm{TeV} /$ nucleon, respectively.

$\mathcal{L}_{N N} \sim \mathcal{O}(1) \mathrm{fb}^{-1}$; see Refs. [17-21]. Similar considerations apply to diffraction in $e \mathrm{~A}$ as to $e p$ collisions. The main difference is the larger contribution from incoherent diffraction $^{3} e+A \rightarrow e+X+A^{*}$ than from coherent diffraction $e+A \rightarrow e+X+A$, the former dominating for $|t|$ larger than a few hundredths of a giga-electron-volt squared. In the following, we focus on coherent diffraction, which could be distinguished from the incoherent case using forward detectors [18].

Assuming the same framework [collinear factorization for hard diffraction, Eq. (7), and Regge factorization, Eq. (9)] described for $e p$ in Secs. II and III A to hold for $e \mathrm{~A}$, nuclear diffractive PDFs (nDPDFs) can be extracted from the diffractive reduced cross sections, Eqs. (4a) and (4b). It should be noted that such nDPDFs have never been measured. With the same electron energy $E_{e}=60 \mathrm{GeV}$ and nuclear beams with $E_{N}=2.76$ and $19.7 \mathrm{TeV} /$ nucleon for the $\mathrm{LHeC}$ and the FCC-eh, respectively, the kinematic coverage is very similar to that shown in Fig. 3.

\footnotetext{
${ }^{3} A^{*}$ denotes a final state in which the nucleus has dissociated to a system of at least two hadrons, but the rapidity gap signature that defines the diffractive event is still present.
}

Due to the lack of previous measurements, there are no parametrizations for nDPDFs, but models exist for the nuclear effects on parton densities defined through the nuclear modification factor

$$
R_{k}^{A}\left(\beta, \xi, Q^{2}\right)=\frac{f_{k / A}^{D(3)}\left(\beta, \xi, Q^{2}\right)}{A f_{k / p}^{D(3)}\left(\beta, \xi, Q^{2}\right)},
$$

with diffractive parton densities in nucleus $A$, $f_{k / A}^{D(3)}\left(\beta, \xi, Q^{2}\right)$. We use the model proposed in Ref. [9], in which parametrizations for nuclear modification factors are provided at the scale $Q^{2}=4 \mathrm{GeV}^{2}$ (extended in $\beta$ and $\xi$ to cover the $\mathrm{LHeC}$ and FCC-eh kinematic regions ${ }^{4}$ ). Then, DGLAP evolution is employed to evolve the ZEUS-SJ proton diffractive PDFs multiplied by $R_{k}^{A}$ from Ref. [9] to obtain the nuclear diffractive PDFs, at any $Q^{2}$. The structure functions and reduced cross sections are then calculated in the same way as in the proton case, and these results are used to obtain the modification factors, analogous to Eq. (15), for these quantities. We have also repeated the calculation in the zero-mass VFNS in order to check that

\footnotetext{
${ }^{4}$ We thank Vadim Guzey for providing them.
} 

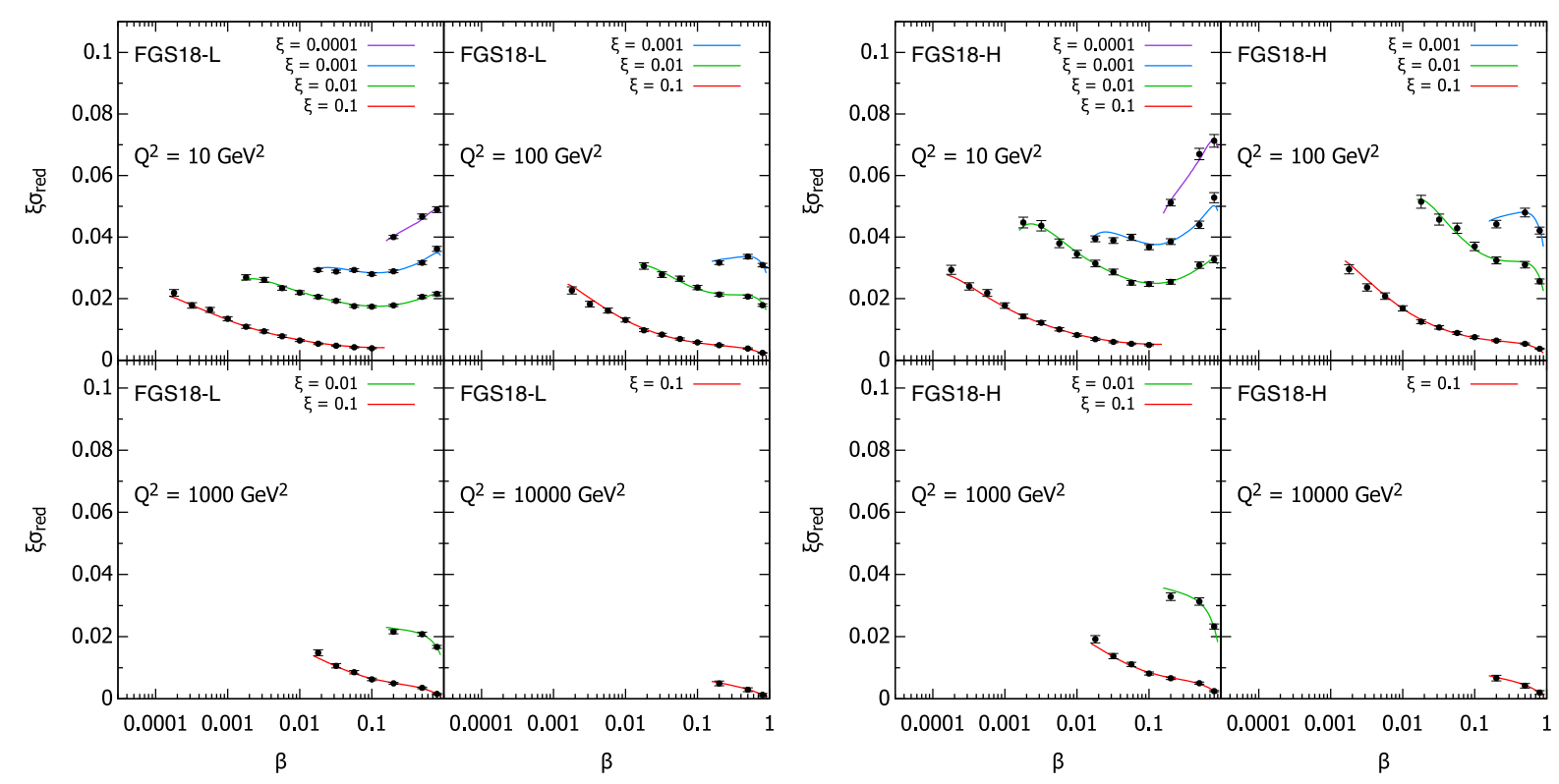

FIG. 14. Simulated data for the diffractive reduced cross section as a function of $\beta$ in bins of $\xi$ and $Q^{2}$ for $e^{208} \mathrm{~Pb}$ collisions at the LHeC, in the models in Ref. [9]. The curves for $\xi=0.01,0.001,0.0001$ are shifted up by $0.01,0.02,0.03$, respectively.

the resulting modification factors do not depend on the applied scheme.

The model in Ref. [9] employs Gribov inelastic shadowing [4], which relates diffraction in $e p$ to nuclear shadowing for total and diffractive $e A$ cross sections. It assumes that the nuclear wave function squared can be approximated by the product of one-nucleon densities, neglects the $t$ dependence of the diffractive $\gamma^{*}$-nucleon amplitude compared to the nuclear form factor, introduces a real part in the amplitudes [46], and considers the color fluctuation formalism for the

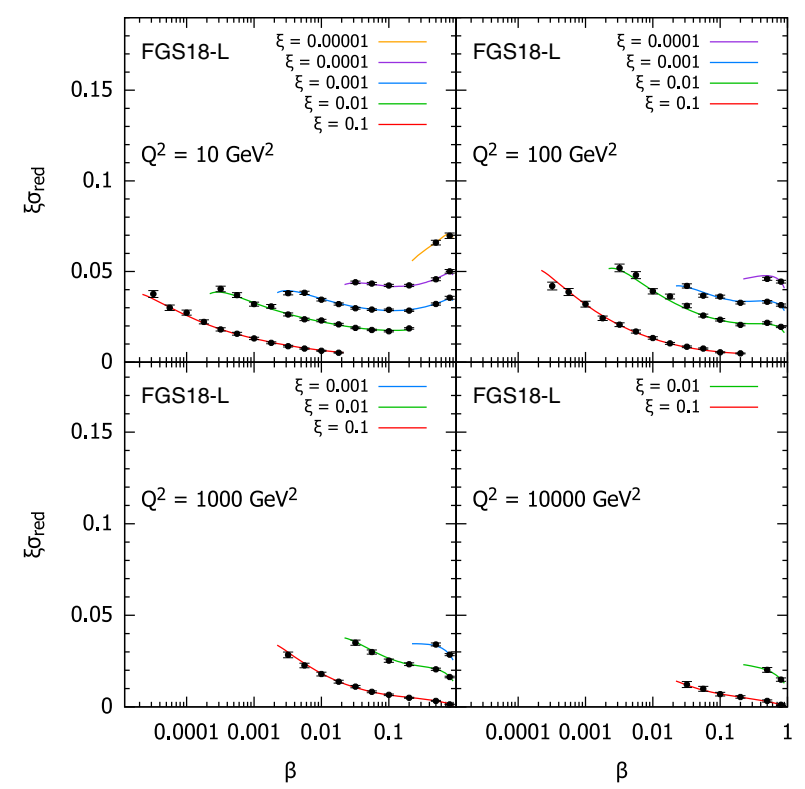

inelastic intermediate nucleon states [47]. There are two variants of the model, named $\mathrm{H}$ and $\mathrm{L}$, corresponding to different strengths of the color fluctuations, giving rise to larger and smaller probabilities for diffraction in nuclei with respect to that in the proton, respectively. To illustrate the results of this model, in Fig. 13, we show the nuclear modification factor, Eq. (15), for $F_{2}^{D(3)}$ and $F_{L}^{D(3)}$ in ${ }^{208} \mathrm{~Pb}$.

Pseudodata were generated using the same method, 5\% uncorrelated systematic error and luminosity $2 \mathrm{fb}^{-1}$ as described for $e p$ in Sec. III C. The results for the $\mathrm{LHeC}$

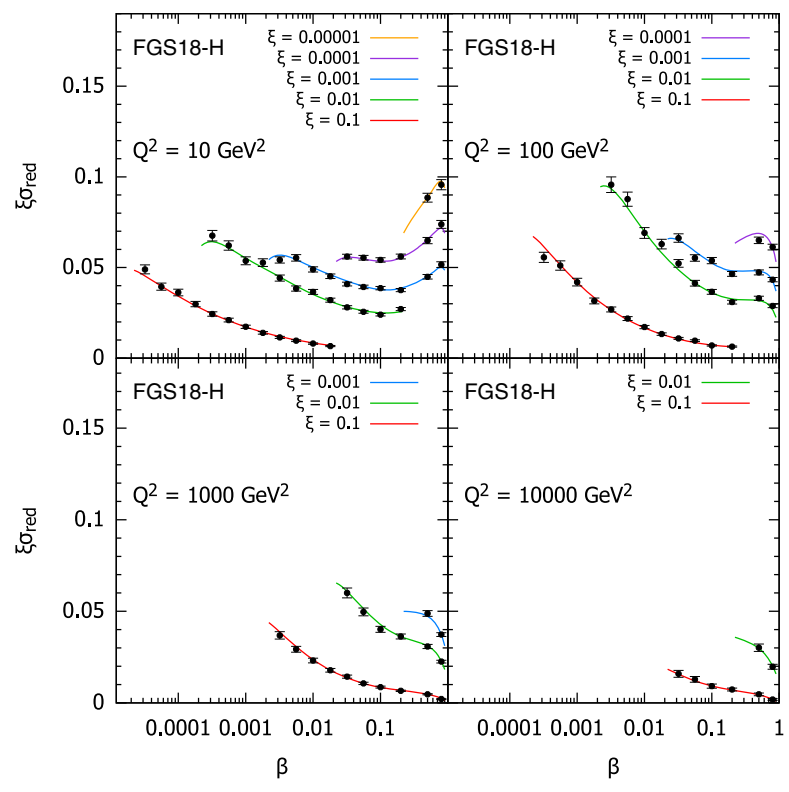

FIG. 15. Simulated data for the diffractive reduced cross section as a function of $\beta$ in bins of $\xi$ and $Q^{2}$ for $e^{208} \mathrm{~Pb}$ collisions at the FCC-eh, in the models in Ref. [9]. The curves for $\xi=0.01,0.001,0.0001,0.00001$ are shifted up by $0.01,0.02,0.03,0.04$, respectively. 

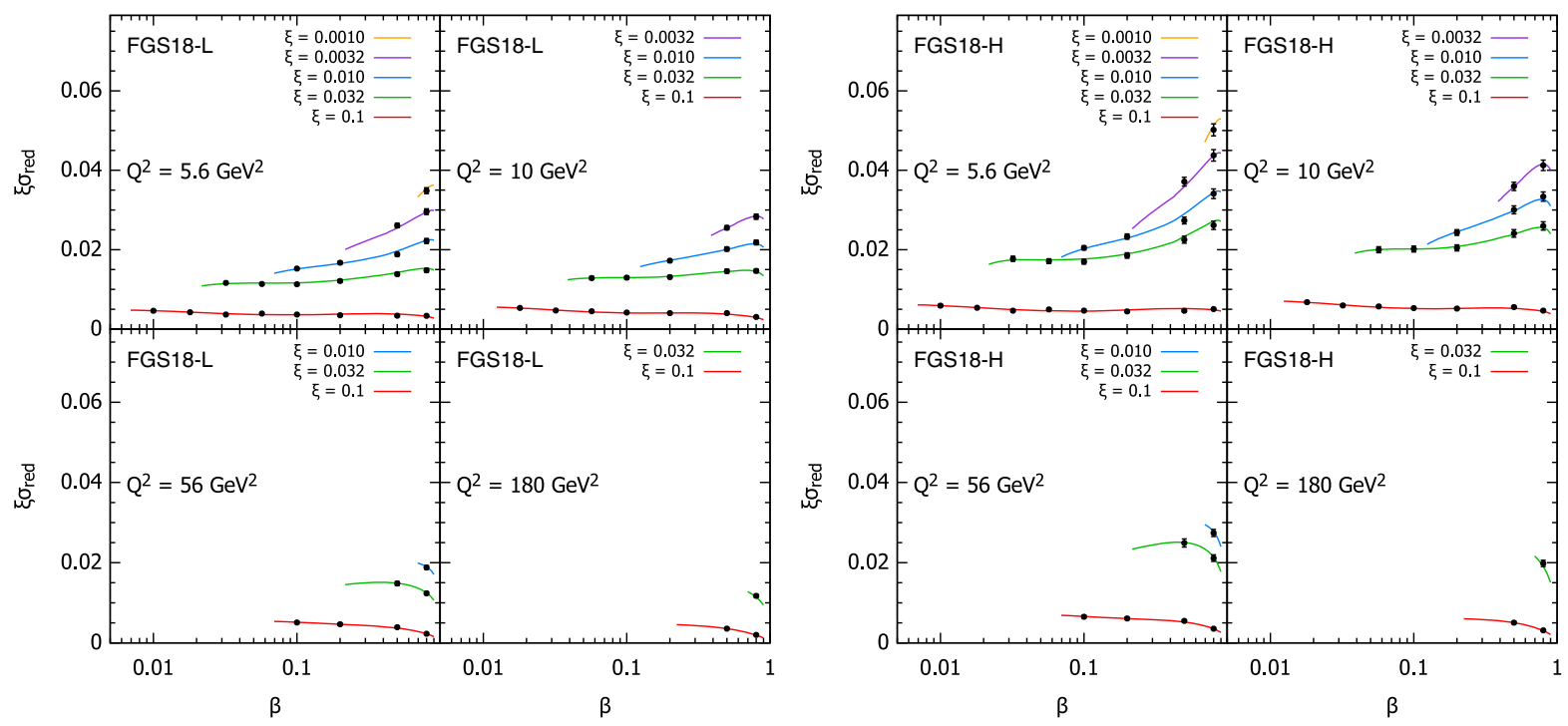

FIG. 16. Simulated data for the diffractive reduced cross section as a function of $\beta$ in bins of $\xi$ and $Q^{2}$ for $e^{197} \mathrm{Au}$ collisions at the EIC, in the models in Ref. [9]. The curves for $\xi=0.032,0.01,0.0032,0.001$ are shifted up by $0.005,0.01,0.015,0.02$, respectively.

and FCC-eh are shown in Figs. 14 and 15, respectively (for a selected subset of bins). The similarly large coverage and small uncertainty (dominated by the assumed systematics) illustrated in these two figures compared to Figs. 6 and 7 make it clear that an accurate extraction of nDPDFs in ${ }^{208} \mathrm{~Pb}$ in an extended kinematic region, similar to that shown in Figs. 8, 9, and 10, will be possible. We also include in Fig. 16 the corresponding results for $e$ Au collisions at the EIC. Studies performed for $e p$ at those energies show that the expected accuracy for the extraction of DPDFs at the EIC is comparable to that in existing DPDFs for the proton at HERA. Assuming, as we did for the LHeC and FCC-eh, a similar experimental uncertainty, integrated luminosity, and kinematic coverage, the accuracy in the extraction of nDPDFs at the EIC would then be similar to that of existing HERA fits.

\section{CONCLUSIONS}

In this paper, we have investigated the potential of the LHeC and FCC-eh machines for the measurement of diffractive cross sections and to constrain the diffractive parton densities. The $\mathrm{LHeC}$ machine would extend the available kinematic range in $x$ by a factor of order 20 and the maximum $Q^{2}$ by a factor of order 100 . The FCC-eh machine would extend the accessible region by an order of magnitude with respect to LHeC both in $x$ and $Q^{2}$. This translates into a range of available $\xi$ down to $10^{-4}$ at the LHeC and down to $10^{-5}$ for FCC-eh for a wide range of $\beta$. With the assumed very conservative integrated luminosity of $2 \mathrm{fb}^{-1}$, we have generated large pseudodata sets of 1200-1800 points for the $\mathrm{LHeC}$ and of 1700-2600 points for the FCC-eh, depending on the minimum $Q^{2}$. The simulated data have very small error bars, dominated by the assumed 5\% systematic error. We have performed fits of the diffractive parton densities to the simulated pseudodata, following the methodology employed previously at HERA. The DPDF determination using the pseudodata substantially improves the precision achieved in the HERA analysis, reducing the DPDF uncertainties by a factor 5-7 for the $\mathrm{LHeC}$ and $10-15$ for the FCC-eh.

We stress that the uncertainty bands shown in the corresponding plots come purely from experimental errors. No attempt is made to evaluate theoretical sources of uncertainty, due, for example, to fixed parameters in the initial conditions or the evolution or to the functional form of the parton parametrization at the starting scale. This corresponds to our aim of establishing the experimental precision achievable in these new machines. Besides, if the luminosity were increased, one could perform a finer binning and constrain the extracted DPDFs even more.

The accuracy of the DPDF extraction depends only mildly on the maximal value of $\xi$. In particular, we found that changing $\xi$ from 0.32 to 0.1 has a negligible impact on the precision of the extracted DPDFs. This is very encouraging since the large $\xi$ region is very challenging experimentally and theoretically. On the other hand, we found a rather large sensitivity to the functional form of the gluon DPDF; specifically, a flat and nonflat gluon-which were indistinguishable at HERA-produce sizeably different $\chi^{2} /$ ndf at the LHeC and FCC-eh. Besides, the fits are also sensitive to the assumed minimal value of $Q^{2}$ used in the DGLAP fits. This feature is understandable since the DGLAP evolution is very sensitive to the low- $Q^{2}$ region, which is crucial for constraining the initial condition. This fact indicates the potential of both machines to constrain the diffractive parton densities in this region and, eventually, physics that goes beyond the standard twist-2 
DGLAP evolution. Finally, we have investigated the possibility of inclusive diffraction in the case of nuclear targets. Using models which employ Gribov inelastic shadowing, we make predictions for the nuclear ratios for the diffractive structure functions $F_{2}$ and $F_{L}$ and provide the simulated datasets. We find that the accurate measurement of the nuclear diffractive cross section would be possible in the nuclear case, with similar coverage in $\beta$, $\xi$, and $Q^{2}$ and precision similar to the proton case.

The extended kinematic range of both machines offers new exciting possibilities in diffraction. One is that they are sensitive to the top contribution to diffraction. Since HERA did not give access to the top, none of the models used to simulate the pseudodata provides a reliable contribution from the top quark. In the present analysis, the top contribution was thus neglected, but it could be investigated in further studies, particularly for the FCC-eh. Furthermore, diffractive dijets could also be included, and their impact on the extraction of DPDFs could be evaluated. Another interesting possibility is that of charged current diffraction. This was measured at HERA, but in a very limited kinematic range and with very small statistics. In future DIS machines, this would certainly be a much better explored process and would provide additional tests for factorization in diffraction.

Summarizing, both the $\mathrm{LHeC}$ and its higher-energy version, the FCC-eh, offer unprecedented capabilities for studying diffraction both in $e p$ and $e A$. This first exploratory study illustrates some of the huge range of opportunities. More extensive studies, both on the phenomenological side and at the detector level, are left for the future. These new possibilities for investigating proton and nuclear structure will eventually open new avenues in the understanding of dynamics beyond linear evolution, such as higher twists and nonlinear effects, and, ultimately, hopefully, confinement.

\section{ACKNOWLEDGMENTS}

We thank Vadim Guzey for providing the FGS parametrization and Max Klein for reading the manuscript. We also thank John Collins and Krzysztof Golec-Biernat for discussions. N. A. was supported by Ministerio de Ciencia e Innovación of Spain under Projects No. FPA2014-58293C2-1-P and No. FPA2017-83814-P and Unidad de Excelencia María de Maetzu under Project No. MDM2016-0692, by Xunta de Galicia (Consellería de Educación) within the Strategic Unit AGRUP2015/11, and by FEDER. This work has been performed in the framework of COST Action CA15213 "Theory of hot matter and relativistic heavy-ion collisions." W. S. was supported by the National Science center, Poland, Grant No. 2014/13/B/ST2/02486. A. M. S. was supported by the Department of Energy Grant No. DE-SC-0002145 as well as the National Science Center, Poland, Grant No. 2015/17/ B/ST2/01838.
[1] C. Adloff et al., Inclusive measurement of diffractive deep inelastic ep scattering, Z. Phys. C 76, 613 (1997).

[2] J. Breitweg et al., Measurement of the diffractive structure function $F_{2}^{\mathrm{D}(4)}$ at HERA, Eur. Phys. J. C 1, 81 (1998).

[3] P. Newman and M. Wing, The Hadronic final state at HERA, Rev. Mod. Phys. 86, 1037 (2014).

[4] V. N. Gribov, Glauber corrections and the interaction between high-energy hadrons and nuclei, Zh. Eksp. Teor. Fiz. 56, 892 (1969) [Sov. Phys. JETP 29, 483 (1969)].

[5] J.C. Collins, Proof of factorization for diffractive hard scattering, Phys. Rev. D 57, 3051 (1998); Erratum, Phys. Rev. D 61, 019902(E) (1999).

[6] A. Berera and D. E. Soper, Behavior of diffractive parton distribution functions, Phys. Rev. D 53, 6162 (1996).

[7] L. Trentadue and G. Veneziano, Fracture functions: An improved description of inclusive hard processes in QCD, Phys. Lett. B 323, 201 (1994).

[8] M. Klasen and G. Kramer, Review of factorization breaking in diffractive photoproduction of dijets, Mod. Phys. Lett. A 23, 1885 (2008).

[9] L. Frankfurt, V. Guzey, and M. Strikman, Leading twist nuclear shadowing phenomena in hard processes with nuclei, Phys. Rep. 512, 255 (2012).
[10] V. Guzey and M. Klasen, Diffractive dijet photoproduction in ultraperipheral collisions at the LHC in next-to-leading order QCD, J. High Energy Phys. 04 (2016) 158.

[11] F. Gelis, E. Iancu, J. Jalilian-Marian, and R. Venugopalan, The color glass condensate, Annu. Rev. Nucl. Part. Sci. 60, 463 (2010).

[12] N. N. Nikolaev and B. G. Zakharov, Color transparency and scaling properties of nuclear shadowing in deep inelastic scattering, Z. Phys. C 49, 607 (1991).

[13] A. H. Mueller, Soft gluons in the infinite momentum wave function and the BFKL pomeron, Nucl. Phys. B415, 373 (1994).

[14] N. N. Nikolaev, B. G. Zakharov, and V. R. Zoller, Unusual effects of diffraction dissociation for multiproduction in deep inelastic scattering on nuclei, Z. Phys. A 351, 435 (1995).

[15] L. L. Frankfurt and M. I. Strikman, Diffraction off nuclei in color singlet models of shadowing, Phys. Lett. B 382, 6 (1996).

[16] H. Kowalski, T. Lappi, C. Marquet, and R. Venugopalan, Nuclear enhancement and suppression of diffractive structure functions at high energies, Phys. Rev. C 78, 045201 (2008). 
[17] J. B. Dainton, M. Klein, P. Newman, E. Perez, and F. Willeke, Deep inelastic electron-nucleon scattering at the LHC, J. Instrum. 1, P10001 (2006).

[18] J. L. A. Fernandez et al., A large hadron electron collider at CERN: Report on the physics and design concepts for machine and detector, J. Phys. G 39, 075001 (2012).

[19] M. Klein, Future deep inelastic scattering with the LHeC, in From My Vast Repertoire...: Guido Altarelli's Legacy, edited by A. Levy, S. Forte, and G. Ridolfi (World Scientific, Singapore, 2018), pp. 303-347.

[20] F. Bordry, M. Benedikt, O. Bruning, J. Jowett, L. Rossi, D. Schulte, S. Stapnes, and F. Zimmermann, Machine parameters and projected luminosity performance of proposed future colliders at CERN, arXiv:1810.13022.

[21] O. Bruning, J. Jowett, M. Klein, D. Pellegrini, D. Schulte, and F. Zimmermann, Future circular collider study FCC-eh baseline parameters, CERN Report No. FCC-ACC-RPT012, 2017.

[22] A. Abada et al., Future circular collider study. Volume 1: Physics opportunities. Conceptual design report, Eur. Phys. J. C 79, 474 (2019).

[23] A. Abada et al., Future circular collider study. Volume 3: The Hadron collider (FCC-hh). Conceptual design report, Eur. Phys. J. Spec. Top. 228, 755 (2019).

[24] A. Accardi et al., Electron ion collider: The next QCD frontier: Understanding the glue that binds us all, Eur. Phys. J. A 52, 268 (2016).

[25] A. Aktas et al., Measurement and QCD analysis of the diffractive deep-inelastic scattering cross-section at HERA, Eur. Phys. J. C 48, 715 (2006).

[26] S. Chekanov et al., Deep inelastic scattering with leading protons or large rapidity gaps at HERA, Nucl. Phys. B816, 1 (2009).

[27] S. Chekanov et al., Study of deep inelastic inclusive and diffractive scattering with the ZEUS forward plug calorimeter, Nucl. Phys. B713, 3 (2005).

[28] A. Aktas et al., Diffractive deep-inelastic scattering with a leading proton at HERA, Eur. Phys. J. C 48, 749 (2006).

[29] S. Chekanov et al., A QCD analysis of ZEUS diffractive data, Nucl. Phys. B831, 1 (2010).

[30] F. D. Aaron et al., Measurement of the cross section for diffractive deep-inelastic scattering with a leading proton at HERA, Eur. Phys. J. C 71, 1578 (2011).

[31] F. D. Aaron et al., Inclusive measurement of diffractive deep-inelastic scattering at HERA, Eur. Phys. J. C 72, 2074 (2012).
[32] V. N. Gribov and L. N. Lipatov, e+ e- pair annihilation and deep inelastic e p scattering in perturbation theory, Yad. Fiz. 15, 1218 (1972) [Sov. J. Nucl. Phys. 15, 675 (1972)].

[33] V.N. Gribov and L. N. Lipatov, Deep inelastic epscattering in perturbation theory, Yad. Fiz. 15, 781 (1972) [Sov. J. Nucl. Phys. 15, 438 (1972)].

[34] G. Altarelli and G. Parisi, Asymptotic freedom in parton language, Nucl. Phys. B126, 298 (1977).

[35] Y. L. Dokshitzer, Calculation of the structure functions for deep inelastic scattering and $\mathrm{e}+\mathrm{e}$ - annihilation by perturbation theory in quantum chromodynamics, Zh. Eksp. Teor. Fiz. 73, 1216 (1977) [Sov. Phys. JETP 46, 641 (1977)].

[36] J. C. Collins and W.-K. Tung, Calculating heavy quark distributions, Nucl. Phys. B278, 934 (1986).

[37] R. S. Thorne and W. K. Tung, PQCD formulations with heavy quark masses and global analysis, arXiv:0809.0714.

[38] J. F. Owens, $Q^{2}$-dependent parametrizations of pion parton distribution functions, Phys. Rev. D 30, 943 (1984).

[39] M. Gluck, E. Reya, and A. Vogt, Pionic parton distributions, Z. Phys. C 53, 651 (1992).

[40] A. Aktas et al., Dijet cross sections and parton densities in diffractive DIS at HERA, J. High Energy Phys. 10 (2007) 042.

[41] F. D. Aaron et al., Combined inclusive diffractive cross sections measured with forward proton spectrometers in deep inelastic ep scattering at HERA, Eur. Phys. J. C 72, 2175 (2012).

[42] R. S. Thorne and R. G. Roberts, Ordered analysis of heavy flavour production in deep inelastic scattering, Phys. Rev. D 57, 6871 (1998).

[43] L. Motyka, M. Sadzikowski, and W. Slominski, Evidence of strong higher twist effects in diffractive DIS at HERA at moderate $Q^{2}$, Phys. Rev. D 86, 111501 (2012).

[44] L. Del Debbio et al. (NNPDF Collaboration), Unbiased determination of the proton structure function $F_{2}^{p}$ with faithful uncertainty estimation, J. High Energy Phys. 03 (2005) 080.

[45] J. Pumplin, D. Stump, R. Brock, D. Casey, J. Huston, J. Kalk, H. L. Lai, and W. K. Tung, Uncertainties of predictions from parton distribution functions. 2. The Hessian method, Phys. Rev. D 65, 014013 (2001).

[46] V. N. Gribov and A. A. Migdal, Properties of the pomeranchuk pole and the branch cuts related to it at low momentum transfer, Yad. Fiz. 8, 1002 (1968) [Sov. J. Nucl. Phys. 8, 583 (1969)].

[47] L. L. Frankfurt, G. A. Miller, and M. Strikman, The geometrical color optics of coherent high-energy processes, Annu. Rev. Nucl. Part. Sci. 44, 501 (1994). 